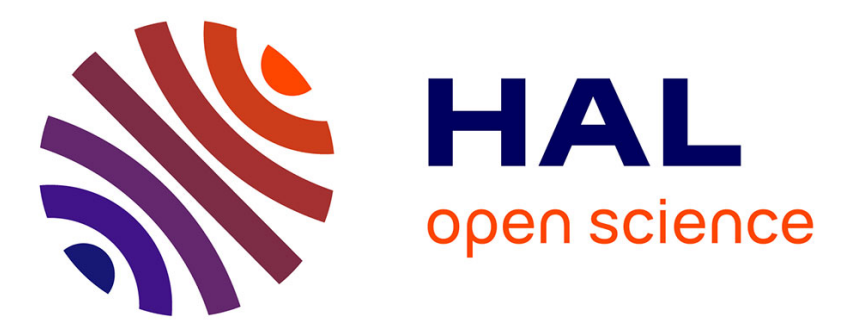

\title{
Initial breakup of a small-diameter liquid jet by a high-speed gas stream
}

\author{
C.M. Varga, Juan C. Lasheras, Emil J. Hopfinger
}

\section{To cite this version:}

C.M. Varga, Juan C. Lasheras, Emil J. Hopfinger. Initial breakup of a small-diameter liquid jet by a high-speed gas stream. Journal of Fluid Mechanics, 2003, 497, pp.405-434. 10.1017/S0022112003006724 . hal-00221378

\section{HAL Id: hal-00221378 \\ https://hal.science/hal-00221378}

Submitted on 7 Apr 2020

HAL is a multi-disciplinary open access archive for the deposit and dissemination of scientific research documents, whether they are published or not. The documents may come from teaching and research institutions in France or abroad, or from public or private research centers.
L'archive ouverte pluridisciplinaire HAL, est destinée au dépôt et à la diffusion de documents scientifiques de niveau recherche, publiés ou non, émanant des établissements d'enseignement et de recherche français ou étrangers, des laboratoires publics ou privés. 


\title{
Initial breakup of a small-diameter liquid jet by a high-speed gas stream
}

\author{
By C. M. VARGA ${ }^{1} \uparrow$, J. C. LASHERA $S^{1}$ AND E. J. HOPFINGER \\ ${ }^{1}$ Department of Mechanical and Aerospace Engineering, University of California, San Diego, \\ La Jolla, CA 92093-0411, USA \\ ${ }^{2}$ LEGI-CNRS/UJF/INPG, BP 53, 38041 Grenoble Cedex, France
}

The situation of a small-diameter liquid jet exposed to a large-diameter high-speed gas jet (gas-to-liquid nozzle area ratio of order 100 to 1000) is investigated experimentally. Flow visualization and particle-sizing techniques are employed to examine the initial jet breakup process and primary liquid atomization. Observations of the initial breakup of the liquid jet in the near-nozzle region, combined with droplet-size measurements, are used in an effort to elucidate the dominant mechanism of primary breakup of the liquid. It is shown that for large aerodynamic Weber numbers, the bulk of the liquid atomization is completed within a few gas-jet diameters of the nozzle exit, inside of the potential cone of the gas flow. Breakup is therefore completed within the zone of constant ambient gas velocity. It is argued that the mechanism of initial jet breakup is similar to that of a liquid drop suddenly exposed to a high-speed gas stream. A phenomenological breakup model is proposed for the initial droplet size, based upon the accelerative, secondary destabilization (via Rayleigh-Taylor instability) of the liquid wave crests resulting from the primary Kelvin-Helmholtz instability of the liquid jet surface. Primary mean droplet sizes are shown to scale well on the most unstable Rayleigh-Taylor wavelength, and the dependence of the droplet diameter on both the atomizing gas velocity and the liquid surface tension are successfully captured by the proposed breakup model.

\section{Introduction}

Liquid jet breakup and atomization by a high-speed gas stream is a complicated multi-parameter two-phase flow problem which resists clear understanding. At present, it is not possible to predict the variation of droplet diameters and their distribution as a function of injection conditions. The recent review by Lasheras \& Hopfinger (2000) of the current state of knowledge of both near-field and far-field breakup mechanisms in gas-assisted liquid atomization highlights the poor understanding and many open questions which remain in this area of fluids research. For combustion applications, many empirical correlations are available for the droplet size as a function of injection parameters (Lefebvre 1989), however, more detailed studies of fundamental breakup mechanisms are clearly needed in order to construct predictive models.

The breakup and atomization of a liquid jet injected into a high-speed gas stream is fundamentally different from that which occurs for the same liquid jet discharging into a stagnant gas. For cases in which the momentum flux of the gas stream is of the

$\dagger$ Present address: Nektar Therapeutics, San Carlos, CA 94070, USA. 


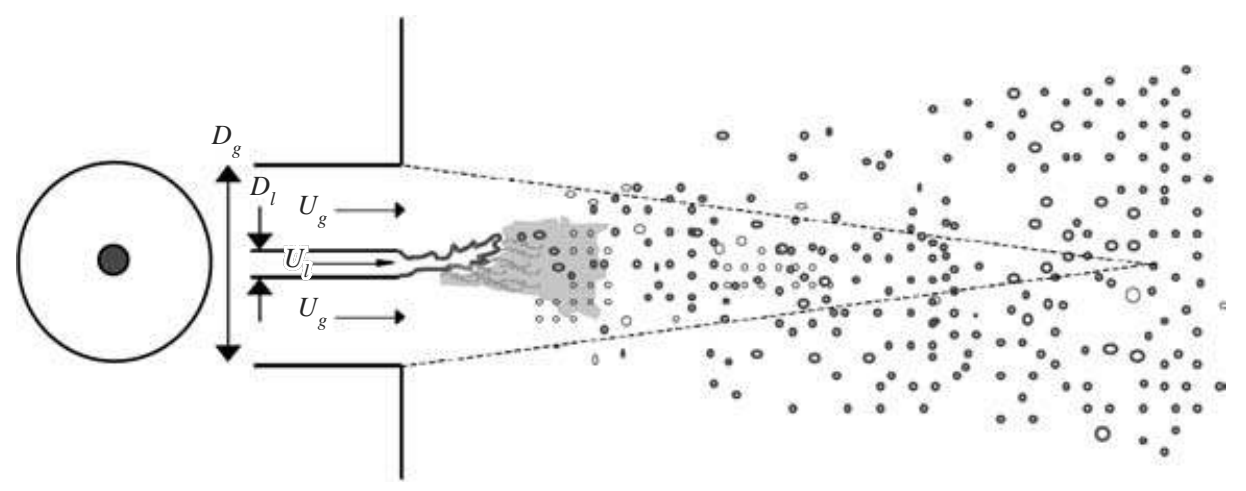

FIGURE 1. Large-area ratio coaxial nozzle and spray schematic.

same order, or exceeds that of the liquid jet, the breakup and atomization is caused by a kinetic energy transfer from the gas to the liquid. This type of atomization is generally referred to as gas-assisted or airblast/air-assisted atomization when air is the atomizing gas (Lefebvre 1989). A common arrangement for this type of atomization, and a generic injector geometry on which many breakup and atomization studies have been based, is the coaxial configuration shown schematically in figure 1 . In this configuration, a central liquid jet of diameter $D_{l}$, and velocity $U_{l}$ is atomized by a high-speed co-flowing annular gas stream with velocity $U_{g}$ and diameter $D_{g}$. Practical applications of coaxial atomization are numerous, particularly in combustion systems. In liquid-propellant rocket engines for example, a large number of coaxial atomizers are employed to supply the fuel and oxidizer for combustion. In each atomizer, a central liquid oxygen (LOX) jet is broken and atomized by a high-speed annular hydrogen gas stream (Burick 1972).

The generally agreed upon earliest work on coaxial atomization was an experimental study by Nukiyama \& Tanasawa (1939) who obtained an expression for the Sauter mean diameter $\left(\mathrm{SMD}=\sum N_{i} d_{i}^{3} / \sum N_{i} d_{i}^{2}\right.$, where $N_{i}$ is the number of droplets per unit volume in size class $i$ and $d_{i}$ is the droplet diameter) as a function of the injection parameters; in particular, they obtained the dependence of the SMD on the atomizing gas velocity, $U_{g}$. Since these early experiments, many other investigations (e.g. Rizkalla \& Lefebvre 1975; Lorenzetto \& Lefebvre 1977) have led to a plethora of empirical expressions for the mean droplet diameters in coaxial jet sprays. A summary of a fair number of these expressions for airblast atomization has been compiled by Lefebvre (1989). The dependence of the primary droplet size, $d$, on the atomizing gas velocity, $U_{g}$, is most often expressed in the form of a power law, $d \propto U_{g}^{-n}$, where $0.7 \leqslant n \leqslant 1.5$. Physical explanations for particular values of the exponent $n$ are generally lacking (Lasheras, Villermaux \& Hopfinger 1998); most investigators have been satisfied by simply determining the best value that fits their data sets, and eventually implement these into practical fluid mechanics and combustion computer codes. This empirical approach, while practical for some purposes, has made it difficult to isolate the effects of individual parameters (Hardalupas \& Whitelaw 1994). Interest in investigating the fundamental physical mechanisms responsible for the dependence of the drop diameter on $U_{g}, U_{l}$, and fluid properties in two-phase coaxial jet flows has not been exploited until recent years.

The instability which occurs at the interface of parallel flowing gas and liquid streams was studied in the mid-twentieth century by G. I. Taylor (Taylor 1963) and has been studied by many others since (e.g. Reitz \& Bracco 1982; Lin \& Lian 1989; 


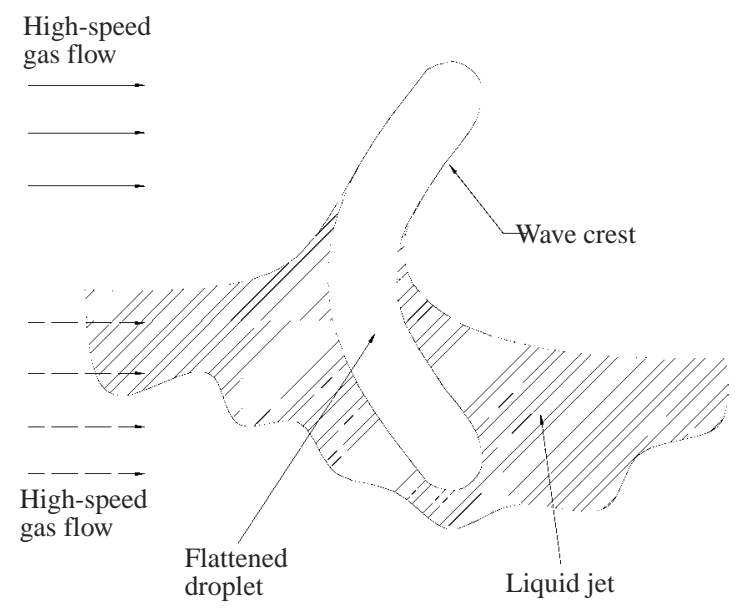

FIGURE 2. Illustration of the analogy between high-speed gas flow over a liquid drop and over a primary wave crest in a coaxial liquid-gas jet.

Mansour \& Chigier 1991; Lin 1995; Raynal 1997; Villermaux 1998). The destabilizing role of a discontinuity in velocity at an interface was first given attention by von Helmholtz (1868), and a detailed analysis of the problem was later performed by Kelvin (1871), including the effects of surface tension and gravity. The wavelength of the most unstable wave developed through the familiar Kelvin-Helmholtz mechanism depends linearly on surface tension, which acts to stabilize small-scale disturbances. This result, however, along with an implied strong dependence on the fast stream velocity, is incompatible with experimental observations (Raynal 1997). The treatment of the interface as an infinitely thin boundary layer (discontinuous interface) has specifically been shown to yield wavelengths which are inconsistent with experimental measurements in coaxial liquid-gas jets (Raynal 1997). The inconsistency stems from the fact that, in practice, the only way to produce shear between parallel flowing streams is to accelerate them in separate channels divided by a rigid boundary. This unavoidable reality leads to the formation of boundary layers which ultimately result in the continuity of the velocity profile at the point where the streams merge, hence introducing a physical length scale.

The analysis of Raynal (1997) showed that the boundary layers formed in the nozzles play a dominant role in determining the wavelength of the interfacial instability, and that this wavelength, $\lambda_{1}$, ultimately depends on the gas vorticity-layer thickness, $\delta_{g}$, and the density ratio as $\lambda_{1} \propto \delta_{g}\left(\rho_{l} / \rho_{g}\right)^{1 / 2}$. Amplification of the instability at the gas/liquid interface leads to the formation of axisymmetric or helical wave sheets which eventually break up into droplets. The mechanisms of formation of these ligaments and droplets, however, are still poorly understood. It is likely that these mechanisms strongly depend on the aerodynamic Weber number, as is known from studies of drop breakup in high-speed gas streams (Pilch \& Erdman 1987; Hsiang \& Faeth 1992; Joseph, Belanger \& Beavers 1999).

The primary wave surface exposed to the oncoming gas flow in the coaxial liquidgas jet is roughly equivalent to half of the windward surface of a liquid drop in a high-speed gas flow. This is illustrated in figure 2 which shows the path of the gas flow over a primary wave crest in a liquid jet, or equivalently, around the drop equator in high-speed drop breakup. A drop placed into a high-speed gas flow initially undergoes a flattening process, and following this, the edges of the drop are drawn out 
at the equator according to Bernoulli's principle and are then subsequently deflected downstream by the high-speed gas. This presents a convex, circular surface to the oncoming gas flow, whose upper hemisphere clearly resembles the liquid sheets that are observed to be drawn out of primary waves in coaxial liquid jet breakup. In the case of jet breakup, an annular area or fractions thereof are exposed to the oncoming gas flow.

Various mechanisms of breakup for low-viscosity liquid droplets subjected to highspeed air streams have been documented by Pilch \& Erdman (1987) as a function of the aerodynamic Weber number, $W e=\rho_{g} U_{g}{ }^{2} D_{l} / \sigma$, where in this case, $D_{l}$ is the drop diameter. For moderate Weber numbers, $12 \leqslant W e \leqslant 100$, surface tension affects the shape of the distorted drop; a cylindrical rim of liquid forms at the equatorial plane as the drop is deformed, and the leading edge retracts because of surface tension. A similar behaviour of rim formation at the wave crests is observed in liquid jet breakup for comparable Weber numbers (Lasheras \& Hopfinger 2000). For We $>100$, Pilch \& Erdman observed that the atomization of the liquid drop occurred by the stripping of sheets from the droplet, and for sufficiently large $W e(W e \geqslant 350)$, by catastrophic destabilization of the entire liquid mass. Hsiang \& Faeth (1992) have also documented various modes of drop breakup by high-speed gas streams as well as the boundaries between these modes, based on the Weber number. Chigier \& Reitz (1995) have pointed out similarities between both the breakup mechanisms and the magnitude of the Weber numbers associated with various breakup regimes for both liquid jet breakup and high-speed drop breakup. This is not surprising when we consider the aforementioned physical similarities which exist between these two flow scenarios (see figure 2).

Joseph et al. (1999) have proposed an accelerative mechanism of destabilization and droplet formation in high-speed drop breakup. They showed that the large accelerations experienced by liquid drops suddenly exposed to high-speed gas streams place them at risk to the well-known Rayleigh-Taylor instability (Taylor 1950). When sufficiently amplified, this instability leads to the destructive breakup of the initial drop into a multitude of small droplets whose sizes tend to scale with the most amplified Rayleigh-Taylor instability wavelength (Joseph et al. 1999). The role of RayleighTaylor waves in drop breakup has also been confirmed by the direct measurements of Hwang, Liu \& Reitz (1996). Acceleration-induced breakup and atomization may also occur in the primary breakup of liquid jets by high-speed coaxial gas streams. Waves drawn out of the liquid by the primary shear instability in coaxial jet flows can experience significant accelerations normal to their surfaces, and thereby become prey to the same Rayleigh-Taylor instability observed in high-speed drop breakup. This mechanism of breakup is particularly plausible in large-momentum-ratio coaxial jets that also possess large gas-to-liquid nozzle exit area ratios. Under these conditions, in addition to causing wave sheet formation, amplification of the primary shear instability can lead to significant excursions of the liquid jet (of small diameter, $D_{l}<\lambda_{1}$ ) from its central axis, providing perpendicular exposure of segments of the liquid surface directly to the high-speed gas stream, and subsequent accelerative destabilization. This instability mechanism is quite different from the Rayleigh-Taylor-type instability due to surface-wave oscillations which has been proposed by Marmottant (2001), and has not been addressed in the context of liquid jet breakup by a high-speed gas stream.

This article reports on an experimental investigation of the initial breakup and atomization process for a small-diameter liquid jet exposed to a large-diameter highspeed gas stream (see figure 1). High-speed video and phase-Doppler particle-sizing techniques were employed to characterize the liquid jet breakup process and the 


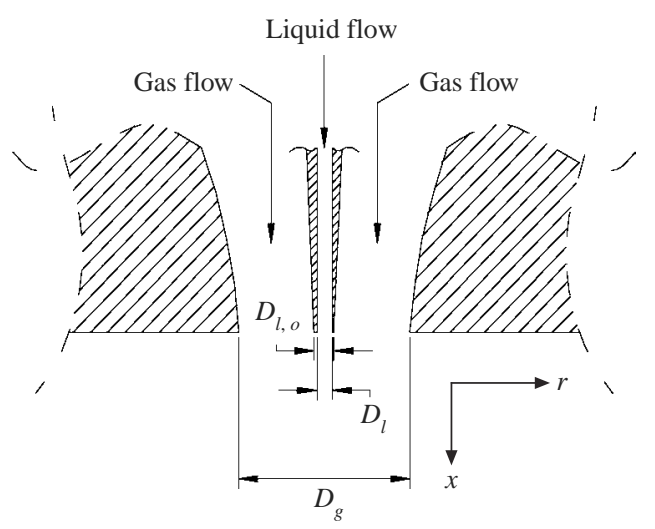

FIGURE 3. Experimental coaxial nozzle configuration, $D_{g}=11.2 \mathrm{~mm}, D_{l}=0.32$ and $1.0 \mathrm{~mm}$, $D_{l, o}=1.3 \mathrm{~mm}$.

resultant droplet-size distribution. These video images and droplet-size measurements were exploited to analyse the physical mechanisms involved in the breakup and atomization of the liquid. In $\S 2$, we describe the experimental configuration and the selected measurement techniques used in this study. In $\S 3$, we present the experimental results including both near-field flow visualizations and drop-size measurements. A phenomenological initial liquid jet breakup model based on the Rayleigh-Taylor instability is developed and presented in $\S 4$, and experimentally observed droplet sizes and jet breakup images are then interpreted within the context of the model. Conclusions of the present investigation are put forward in $\S 5$.

\section{Experimental set-up and methods}

\subsection{Experimental facility and flow generation}

The flow configuration chosen for this study is shown schematically in figure 3 , consisting of the geometrically simple case of a round liquid jet surrounded by a co-flowing annular gas stream. This fundamentally simple geometry provides for well-known nozzle exit conditions and avoids the complicated internal flows that are common to most practical atomizers. The atomization rig used in the current experiments was a modular design, consisting of an upper and lower block structure. The upper section of this block design houses an interchangeable liquid injection tube, while the lower block incorporates four gas injection ports to supply the atomization gas, a distribution chamber, and terminates with a specific gas nozzle. The nozzle-specific lower block can be exchanged to incorporate different gas nozzle configurations. Two different nozzle geometries were chosen for the coaxial gas flow in this investigation. The first nozzle was a smooth convergence nozzle with a contraction ratio of 12:1, while the second nozzle consisted of a straight channel geometry with the same exit dimension. The former provided for a flat velocity profile at the exit with very thin laminar boundary layers, while the latter produced thicker turbulent boundary layers. The selected nozzle diameters for the liquid jet were $D_{l}=1.0 \mathrm{~mm}$ and $D_{l}=0.32 \mathrm{~mm}$, while the outer liquid tube diameter was fixed at $D_{l, o}=1.3 \mathrm{~mm}$. The lip thickness of the liquid injector tube was therefore $0.15 \mathrm{~mm}$ when $D_{l}=1.0 \mathrm{~mm}$ and approximately $0.5 \mathrm{~mm}$ for the smaller nozzle of dimension $D_{l}=0.32 \mathrm{~mm}$. The gas boundary layer is expected to attach to the liquid surface at $x \approx 7\left(D_{l, o}-D_{l}\right) / 2$, so that the slightly 


$\begin{array}{lccc}\quad \text { Fluid } & \text { Density }\left(\mathrm{kg} \mathrm{m}^{-3}\right) & \text { Viscosity }(\mathrm{Pa} \mathrm{s}) & \text { Surface tension }\left(\mathrm{N} \mathrm{m}^{-1}\right) \\ \text { Air } & 1.2 & 1.8 \times 10^{-5} & - \\ \text { Water } & 998 & 1.0 \times 10^{-3} & 0.070 \\ \text { Ethanol } & 791 & 1.2 \times 10^{-3} & 0.023\end{array}$

Table 1. Properties of air, water and ethanol at $20^{\circ} \mathrm{C}$.

larger lip thickness in the case of $D_{l}=0.32 \mathrm{~mm}$ will lead to a longer attachment length. This effect may lead to a slightly larger effective gas boundary-layer thickness for the smaller nozzle. The liquid flow was supplied through a long straight tube of length $60 \mathrm{~mm}$, so that the condition at the outlet was a developed pipe flow. The gas nozzle exit diameter for both the convergent and straight-channel nozzles was $D_{g}=11.2 \mathrm{~mm}$, which yielded an annular gap width of $4.95 \mathrm{~mm}$ and gas-to-liquid exit area ratios of 125 and 1200 for the two liquid nozzles, respectively.

The atomizer assembly was mounted on a vertically oriented two-dimensional computer-controlled traverse. This allowed for the spray to be moved in both the axial $(x)$ and transverse $(r)$ directions with respect to a laboratory-fixed measurement control volume. A receiving reservoir outfitted with absorbent material was placed downstream to collect the spray and to prevent any recirculation of liquid droplets into the measurement volume. Suction fans placed around the perimeter of the damping reservoir were used to remove the spray and to further reduce the possibility of measurement contamination. Flow measuring equipment was affixed to horizontal rails surrounding the atomization rig. Data acquisition and movement of the coaxial atomizer in the two-dimensional $(x, r)$-plane were controlled remotely through an isolated computer workstation.

The fluids chosen for the current study were air for the gas flow, and both water and ethanol for the liquid flow. Relevant properties of these three fluids are given in table 1. The gas flow was supplied by a compressed-air source and metered using a combination of two precision rotameters. The water and ethanol flows were supplied centrally through the top of the atomizer test rig, and the liquid volume flow rates were carefully monitored via three precision flow meters. Section-averaged liquid and gas nozzle exit velocities, $U_{l}$ and $U_{g}$, were determined through their respective volume flow rates and exit areas, $A_{l}$ and $A_{g}$. The section-averaged air velocity, $U_{g}$, was varied from $30 \mathrm{~m} \mathrm{~s}^{-1}$ to $165 \mathrm{~m} \mathrm{~s}^{-1}$, and the liquid velocity, $U_{l}$, was varied from $1.7 \mathrm{~m} \mathrm{~s}^{-1}$ to $16.6 \mathrm{~m} \mathrm{~s}^{-1}$. All experiments were carried out at atmospheric pressure.

\subsection{Measurement techniques}

Two measurement techniques were employed for the characterization of the liquid breakup process in this study. Flow visualizations were conducted using a high-speed video imaging system to provide both qualitative characteristics and quantitative measures of the liquid flow in the near field, while phase-Doppler anemometry (PDA) was employed just downstream of the initial liquid breakup region to measure droplet sizes. Images of the near-field initial breakup region were captured using a high-speed digital video camera (Photron Super 10K Fastcam) operating at 1000 frames/second. A Kodak Ektapro stroboscopic light source synchronized with the framing rate of the camera was used to back-light the liquid jet/spray for the video imaging. The resolution of the high-speed digital video images was $20 \mu \mathrm{m}$ per pixel. A commercially available phase-Doppler particle analyser (PDPA) from TSI Inc. was used to measure 


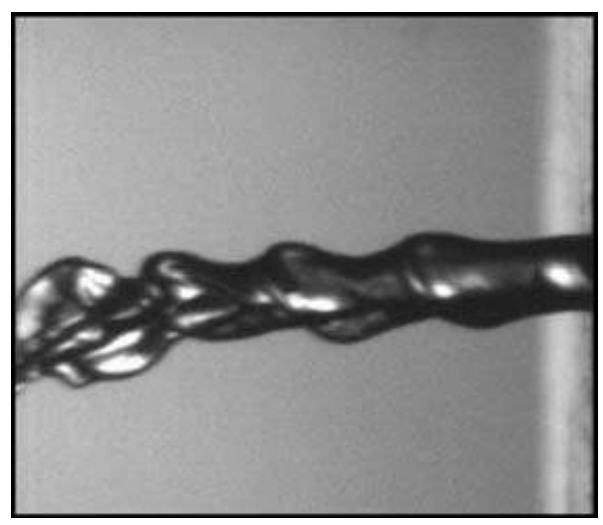

FIGURE 4. Instantaneous initial breakup image, $D_{l}=1.0 \mathrm{~mm}, U_{g}=40 \mathrm{~m} \mathrm{~s}^{-1}, U_{l}=1.7 \mathrm{~m} \mathrm{~s}^{-1}$, $W e=24, R e_{g}=2.6 \times 10^{4}, R e_{l}=1700, m=0.29, M=0.7$.

the drop-size distributions along the centreline of the jet at locations ranging from $x / D_{g}=5$ to $x / D_{g}=60$. At each measurement location in the spray, 150000 samples were acquired to ensure accurate statistics.

\section{Results}

\subsection{Relevant non-dimensional groups}

In coaxial liquid-gas jets, there are several non-dimensional parameters which govern the behaviour of both the breakup process and the ensuing two-phase flow. A Reynolds number can be defined for each of the two phases. For the gas, we write $R e_{g}=U_{g}\left(D_{g}-D_{l, o}\right) / v_{g}$, and for the liquid jet, $R e_{l}=U_{l} D_{l} / v_{l}$. A defining parameter for the current experiments is the gas-to-liquid nozzle cross-sectional area ratio

$$
A_{g l}=\frac{D_{g}^{2}-D_{l, o}^{2}}{D_{l}^{2}},
$$

which was made very large for this study, ranging from approximately 125 to 1210 . Another key non-dimensional group, and arguably the most important one in the initial liquid jet breakup process, is the aerodynamic Weber number

$$
W e=\frac{\rho_{g}\left(U_{g}-U_{l}\right)^{2} D_{l}}{\sigma},
$$

which defines the ratio between the destabilizing dynamic pressure forces exerted by the gas on the liquid, and the confining forces associated with the surface tension, $\sigma$. Three other parameters which are relevant to the breakup and atomization of the liquid jet include the gas-to-liquid dynamic pressure ratio, $M=\rho_{g} U_{g}^{2} / \rho_{l} U_{l}^{2}$, the mass flux ratio, $m=\rho_{l} U_{l} / \rho_{g} U_{g} A_{g l}$, and the Ohnesorge number, $O h=\mu_{l} /\left(\rho_{l} D_{l} \sigma\right)^{1 / 2}$, which is the ratio of viscous forces to surface tension forces. In the current study, $O h \sim 10^{-3}$, so that viscous forces do not inhibit breakup.

\subsection{Observations of the initial breakup process}

High-speed video images of the initial coaxial jet breakup process at various representative flow conditions are shown in figures 4-10 for water. The right-hand side of each image corresponds to the nozzle exit plane, and the flow direction is therefore 


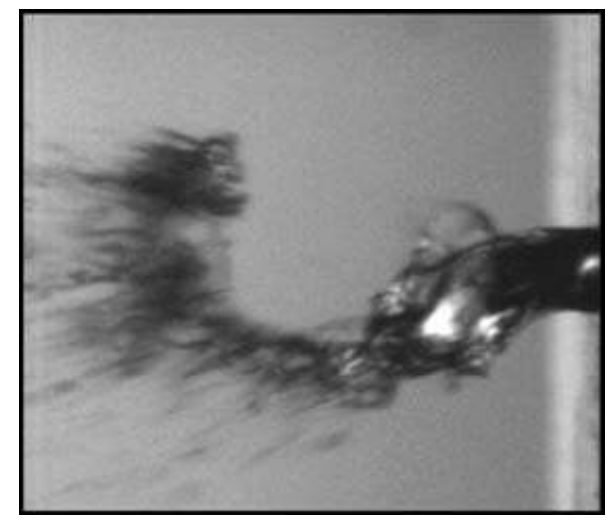

FIGURE 5. Instantaneous initial breakup image, $D_{l}=1.0 \mathrm{~mm}, U_{g}=69 \mathrm{~m} \mathrm{~s}^{-1}, U_{l}=1.7 \mathrm{~m} \mathrm{~s}^{-1}$, $W e=74, R e_{g}=4.6 \times 10^{4}, R e_{l}=1700, m=0.17, M=1.9$.

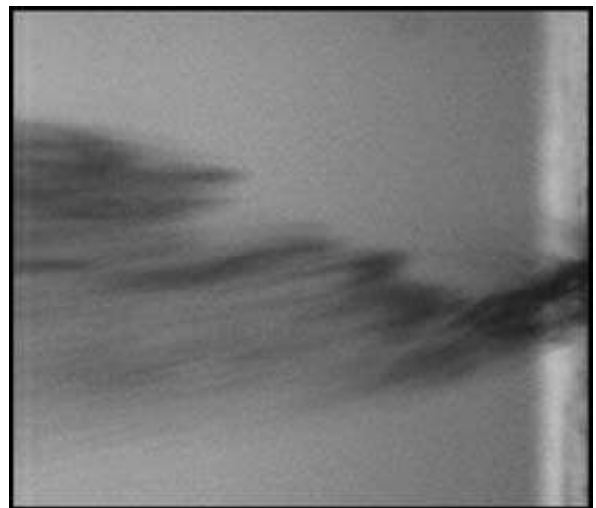

FIGURE 6. Instantaneous initial breakup image, $D_{l}=1.0 \mathrm{~mm}, U_{g}=165 \mathrm{~m} \mathrm{~s}^{-1}, U_{l}=1.7 \mathrm{~m} \mathrm{~s}^{-1}$, $W e=437, R e_{g}=1.1 \times 10^{5}, R e_{l}=1700, m=0.07, M=11.1$.

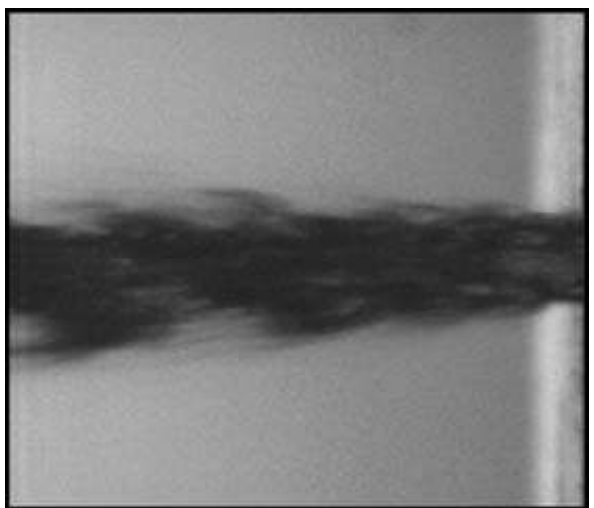

FIGURE 7. Instantaneous initial breakup image, $D_{l}=1.0 \mathrm{~mm}, U_{g}=165 \mathrm{~m} \mathrm{~s}^{-1}, U_{l}=14.6 \mathrm{~m} \mathrm{~s}^{-1}$, $W e=371, R e_{g}=1.1 \times 10^{5}, R e_{l}=1.5 \times 10^{4}, m=0.61, M=0.15$.

from right to left in all cases. In the experiments, the jets were directed downwards, along the axis of gravity. The acceleration due to gravity of the liquid jet increases its velocity by $\Delta U_{l}=\sqrt{2 g x} \leqslant 0.3 \mathrm{~m} \mathrm{~s}^{-1}$. This has been neglected in the analysis of $\S 4$. 


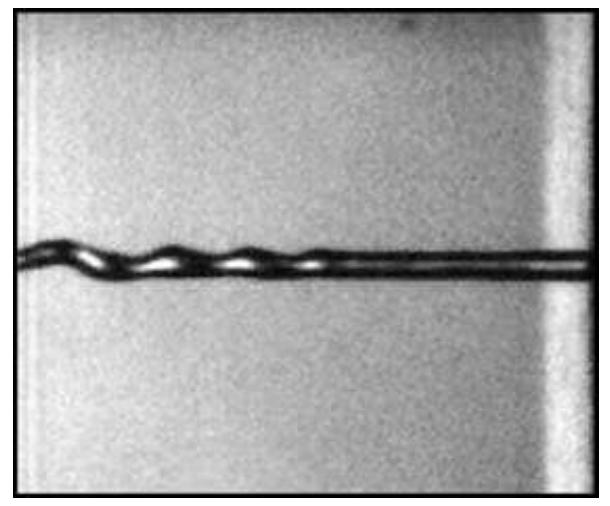

FIGURE 8. Instantaneous initial breakup image, $D_{l}=0.32 \mathrm{~mm}, U_{g}=40 \mathrm{~m} \mathrm{~s}^{-1}, U_{l}=4.9 \mathrm{~m} \mathrm{~s}^{-1}$, $W e=6, R e_{g}=2.6 \times 10^{4}, R e_{l}=1568, m=0.09, M=0.08$.

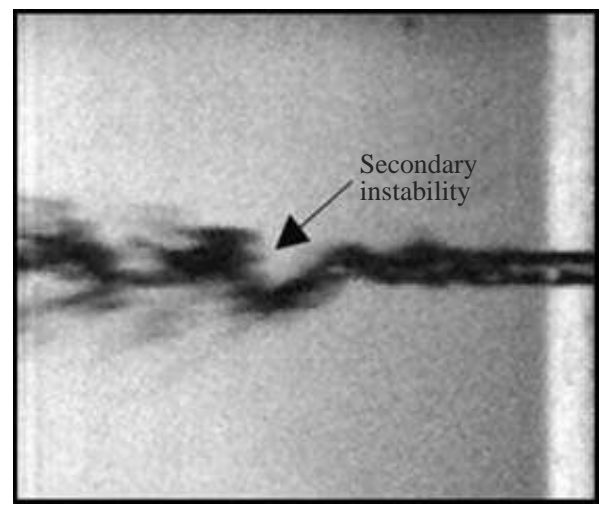

Figure 9. Instantaneous initial breakup image, $D_{l}=0.32 \mathrm{~mm}, U_{g}=100 \mathrm{~m} \mathrm{~s}^{-1}$, $U_{l}=16.5 \mathrm{~m} \mathrm{~s}^{-1}, W e=37, R e_{g}=6.6 \times 10^{4}, R e_{l}=5280, m=0.12, M=0.04$.

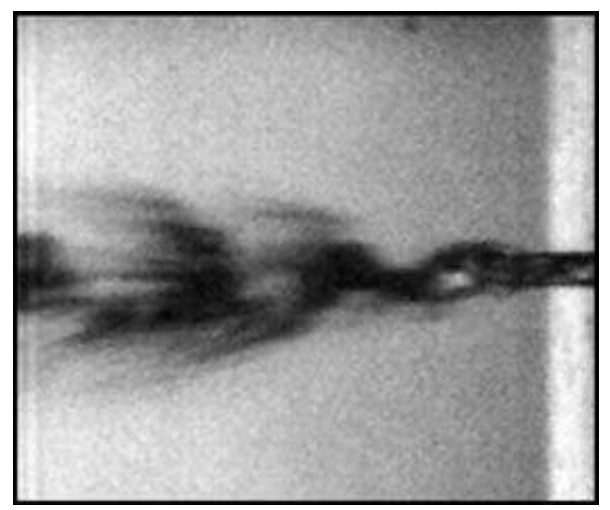

FIGURE 10. Instantaneous initial breakup image, $D_{l}=0.32 \mathrm{~mm}, U_{g}=165 \mathrm{~m} \mathrm{~s}^{-1}$, $U_{l}=16.5 \mathrm{~m} \mathrm{~s}^{-1}, W e=116, R e_{g}=1.1 \times 10^{5}, R e_{l}=5280, m=0.07, M=0.12$.

The downstream extent of these high-speed video images is approximately one gas-jet diameter, and thus, the issuing liquid jet and the visible breakup events observable in these images are entirely within the potential cone of the gas jet, which is a 
zone of constant ambient gas velocity. Figure 4 is an image of the $1.0 \mathrm{~mm}$ water jet issuing at $1.7 \mathrm{~m} \mathrm{~s}^{-1}$ inside an annular gas flow $\left(D_{g}=11.2 \mathrm{~mm}\right)$ with an exit velocity of $40 \mathrm{~m} \mathrm{~s}^{-1}$. The liquid flow is laminar under these conditions $\left(R e_{l}=1700\right)$, and the Weber number is equal to 24 , which places the coaxial jet near the lower boundary of the so-called 'membrane breakup' regime (see Lasheras \& Hopfinger 2000). Membranes are observed to develop just as the liquid jet reaches the left-hand edge of the image. A well-defined wavelength of the primary shear instability (here of helical nature) is evident in this image. Figure 5 presents the coaxial jet for the same liquid Reynolds number as figure 4, but for a larger gas velocity of $69 \mathrm{~m} \mathrm{~s}^{-1}$, which yields $W e=74$ and a larger dynamic pressure ratio $(M=1.9)$. According to the widely accepted breakup regimes for coaxial jets (Faragò \& Chigier 1992; Lasheras \& Hopfinger 2000), the breakup process in this image is at the boundary of membrane breakup. Membranes are clearly visible in figure 5, however, the liquid jet also exhibits smallerscale corrugations and fibres which break apart into droplets. It is to be expected that the breakup regime boundaries depend somewhat on the nozzle geometry, and more specifically on $\lambda_{1}$ with respect to $D_{l}$. The liquid jet is additionally observed to make rather large radial excursions from the central axis, exposing segments of the jet to perpendicular contact with the oncoming gas flow. In figure 6, which corresponds to $W e=437$, the breakup process is much more intense, and the integrity of the liquid jet is compromised much closer to the nozzle exit. The liquid jet is effectively reduced to a fine mist over the very short distance corresponding to the lateral extent of the image. The breakup process in this image appears to be a strong stripping-type breakup which occurs on an intermediate scale associated with a secondary wave structure along the amplified primary instability wavelength. Figure 7 is distinctly different from the preceding images owing to a larger liquid mass loading effect. The liquid velocity in this image is $U_{l}=14.6 \mathrm{~m} \mathrm{~s}^{-1}$, which contributes to a liquid-to-gas mass flux ratio of $m=0.61$ and a dynamic pressure ratio of $M=0.15$. The increased mass flux and momentum of the liquid make it more difficult for the gas flow to instigate the global breakup of the jet. Therefore, the observed breakup is confined to the surface layer of the intact liquid jet which may now penetrate much farther downstream before being broken completely. The production of droplets at the liquid surface in these figures appears to occur through the aerodynamic destabilization of wave crests formed from the primary shear instability. Figure 8 is an image of the $D_{l}=0.32 \mathrm{~mm}$ water jet issuing inside of the same diameter gas jet $\left(D_{g}=11.2 \mathrm{~mm}\right)$. The wavelength, $\lambda_{1}$, of the primary interfacial instability is shown clearly in this highspeed video image with a laminar liquid jet flow and a small Weber number $(W e=6)$. Figure $9\left(D_{l}=0.32 \mathrm{~mm}, W e=37\right)$ provides a very clear illustration of the existence of two distinct instability wavelengths associated with the liquid jet breakup process. The secondary instability can be clearly seen in this image on the third segment of the primary instability wavetrain. The wavelength of this secondary instability appears to be very regular and it is noteworthy that the surface of the liquid segment on which this instability is developing is nearly perpendicular to the direction of the gas stream. Figure 10, with $W e=116$, illustrates the aerodynamic enhancement of this dual-instability breakup mechanism, with a wave disintegration and stripping process which again rapidly reduces the liquid to a fine mist. The breakup in figures 9 and 10 is clearly of the fibre-type, while the Weber numbers calculated with $D_{l}$ are in the ligament and membrane regimes. In these cases, $D_{l}$ has been reduced to $0.32 \mathrm{~mm}$, reducing the Weber number by a factor of three compared with the images of figures 5 and 6 . The wavelength, $\lambda_{1}\left(\propto \delta_{g}\right)$, which is the relevant length scale (as will be seen later), depends on $U_{g}$ only, and has not changed. 


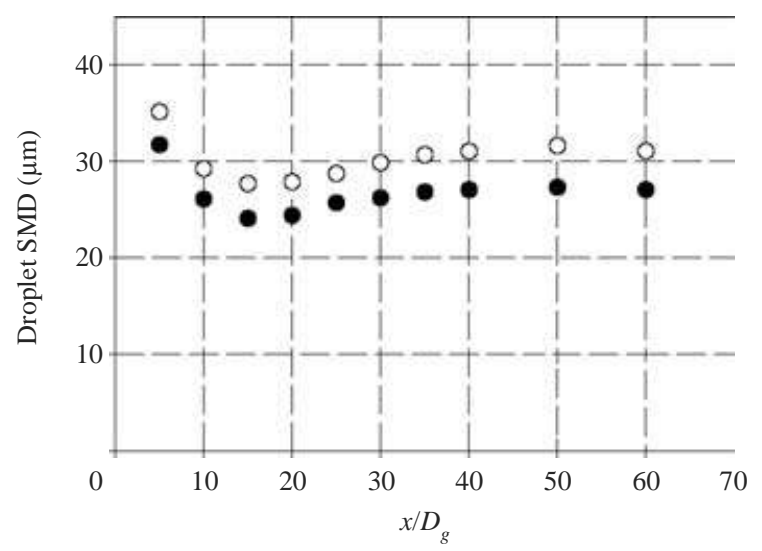

FIGURE 11. Droplet SMD as a function of $x / D_{g}$ for varying liquid jet diameter:

•, $D_{l}=1.0 \mathrm{~mm} ; \bigcirc, 0.32 \mathrm{~mm} ; U_{g}=165 \mathrm{~m} \mathrm{~s}^{-1}, m=0.07$.

\subsection{Droplet-size measurements}

In this subsection, results of droplet-size measurements taken at various locations downstream of the initial breakup region are presented, with a particular focus on the region just downstream of the primary breakup region. Together with the high-speed video observations of the primary breakup process contained in $\S 3.2$, the data presented here serve to aid in determining the most plausible liquid breakup mechanism which occurs in the near field of the large-area ratio two-phase coaxial jet. By varying flow conditions and fluid properties, an attempt was made to determine the dependency of the Sauter mean droplet diameter (SMD) on various quantities. The SMD was chosen as the representative moment of the measured droplet-size distributions for all comparisons in the present study. This diameter represents the liquid volumeto-surface area ratio of the spray and is relevant to the characterization of liquid breakup and atomization processes which generally involve rapid surface generation from an initially compact liquid volume. The SMD is typically representative of the largest size droplets in the drop-size distributions of coaxial jet sprays, and thus is well-suited to comparisons with breakup models which predict maximum stable diameters. All measured droplet-size distributions in the present experiments were unimodal in nature. It should also be noted that because the liquid jet diameter is small in the present experiments, there are large spiral excursions, and distinctions between breakup off-centre and at the centre cannot be made. The radial droplet-size distributions are nearly flat in this case, and centreline droplet-size measurements are therefore expected to be indicative of the overall breakup process.

The sensitivities of both the breakup process and the mean droplet diameter to changes in the liquid jet diameter and the gas nozzle geometry were also explored. The sensitivity of the SMD to the liquid jet diameter for constant mass flux ratio $(m=0.07)$ is shown in figure 11 , which is a plot of the droplet SMD as a function of $x / D_{g}$ for water flow from two different liquid nozzle diameters $\left(D_{l}=1.0 \mathrm{~mm}\right.$ and $D_{l}=0.32 \mathrm{~mm}$ ) and $U_{g}=165 \mathrm{~m} \mathrm{~s}^{-1}$. This data clearly indicates that the mean droplet size is not very sensitive to the liquid jet diameter, which was reduced by a factor of approximately three, and yielded a change in the SMD of only a few microns. The trend is actually opposite to intuition; the droplet-size is observed to be slightly larger for the case of the smaller liquid nozzle diameter. This effect is attributed to the slightly longer gas boundary-layer attachment length for $D_{l}=0.32 \mathrm{~mm}$, which 


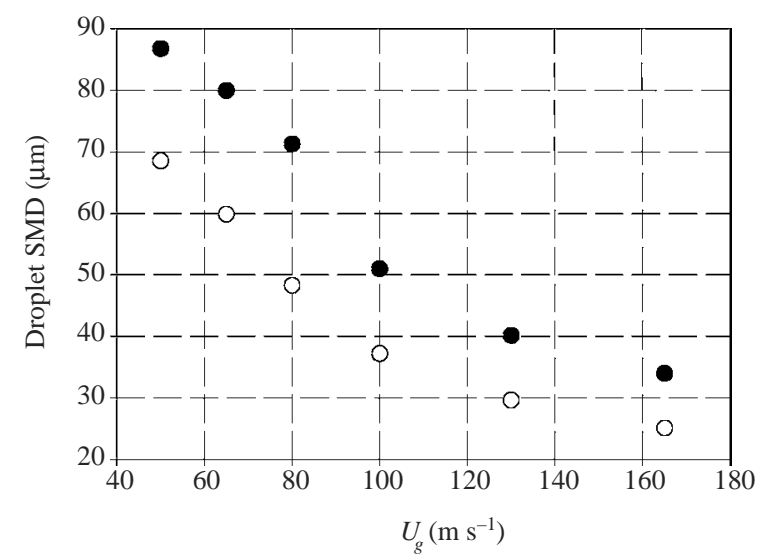

FIGURE 12. Droplet SMD as a function of $U_{g}$ for $\bigcirc$, convergent and $\bullet$, straight injector gas nozzles, $D_{l}=1.0 \mathrm{~mm}, U_{l}=1.7 \mathrm{~m} \mathrm{~s}^{-1}, x / D_{g}=15$.

was discussed in $\S 2.1$. It should also be noted that when $D_{l}$ is smaller, the gas-liquid relative velocity for constant mass flux ratio is accordingly reduced, which is also consistent with the observed trend in the droplet-size. Figure 12 shows a comparison of the droplet SMD as a function of the gas exit velocity at $x / D_{g}=15$ for the two different gas nozzle configurations considered in this study, which were described in $\S 2$. The liquid diameter for this set of experiments was fixed at $D_{l}=1.0 \mathrm{~mm}$ and the liquid jet exit velocity was $U_{l}=1.7 \mathrm{~m} \mathrm{~s}^{-1}$. A significant reduction in the Sauter mean droplet diameter is observed for the convergent gas nozzle, which produces an accelerating flow with thin laminar boundary layers at the nozzle exit, compared to that observed for the straight injector nozzle, which contains thicker turbulent boundary layers.

The effect of liquid surface tension on the mean size of droplets was investigated in the current study by comparing SMD measurements for the breakup of water and ethanol. Ethanol has a surface tension value which is approximately three times less than that of water, while possessing comparable density and viscosity values. A comparison of the properties of these two liquids was presented in $\S 2$ (see table 1). Figure 13 contains plots of droplet SMD as a function of $x / D_{g}$ for both water and ethanol jets emanating from the liquid tube of diameter $D_{l}=0.32 \mathrm{~mm}$. The nozzle exit velocity for both liquids was $U_{l}=16 \mathrm{~m} \mathrm{~s}^{-1}$ and the gas exit velocity was $U_{g}=165 \mathrm{~m} \mathrm{~s}^{-1}$. Significantly smaller mean droplet diameters are observed for ethanol compared to water, and moreover, the size reduction across the measurement range shown in figure 13 appears to be approximately a constant factor. Figure 14 presents a similar comparison for water and ethanol SMDs as a function of the atomizing gas velocity at the downstream location $x / D_{g}=15$. A clear reduction in drop sizes is again observed for the ethanol compared to the water at all gas velocities. In the present experiments, ethanol droplets have been estimated to undergo at most a $5-10 \%$ evaporation between $x / D_{g}$ of order 2 to 3 and $x / D_{g}=15$. This is supported by the measurements of figure 13 which show that the decreases in the SMD between $x / D_{g}=5$ and $x / D_{g}=15$ of the water and ethanol droplets are very similar. Further downstream, the variations with $x$ begin to diverge as evaporation of the ethanol droplets becomes increasingly more important. 


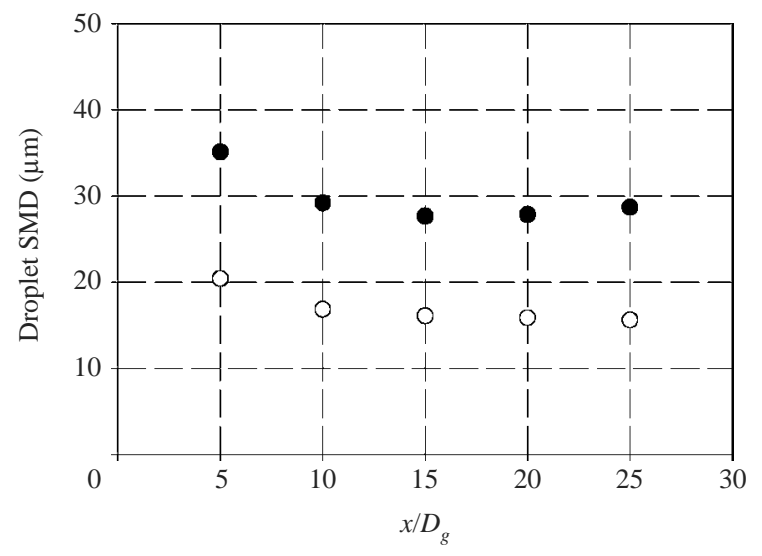

FIGURE 13. Droplet SMD as a function of $x / D_{g}$ for $\bullet$, water and $\bigcirc$, ethanol, $D_{l}=0.32 \mathrm{~mm}$, $U_{l}=16 \mathrm{~m} \mathrm{~s}^{-1}, U_{g}=165 \mathrm{~m} \mathrm{~s}^{-1}$.

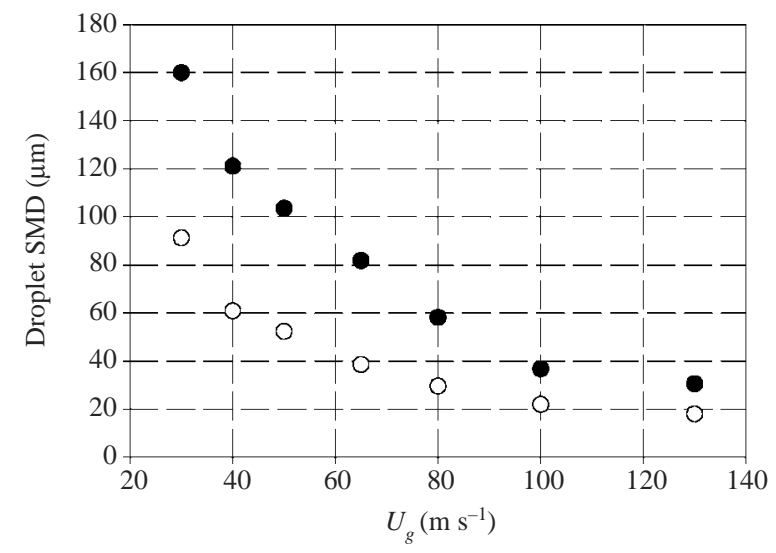

FIgURE 14. Droplet SMD as a function of $U_{g}$ for $\bullet$, water and $\bigcirc$, ethanol, $D_{l}=0.32 \mathrm{~mm}$, $U_{l}=5 \mathrm{~m} \mathrm{~s}^{-1}, x / D_{g}=15$.

\subsection{Drop-size scaling}

The key role played by the aerodynamic Weber number in characterizing the various breakup regimes associated with liquid jet breakup by a coaxial gas stream motivated an effort to reduce the present droplet-size data according to this parameter. A potential scaling for the droplet diameter with the aerodynamic Weber number was inspired by the results presented in figure 13 . Figure 15 contains a plot of the ratio of the water SMD to the ethanol SMD from the data of figure 13. This ratio appears to be very close to $\sqrt{\sigma_{\text {water }} / \sigma_{\text {ethanol }}} \approx \sqrt{3}$ (shown as a dotted line in figure 15 ), which suggests the scaling $d \propto W e^{-1 / 2}$. Figure 16 contains five distinct droplet-size data sets which include measurements at $x / D_{g}=15$ for both water and ethanol, two different liquid nozzle diameters, and various liquid nozzle exit velocities, all plotted as a function of $W e^{1 / 2}$. The data collapse very well to two distinct curves, one corresponding to each liquid jet diameter, $D_{l}$. The results of figure 11 suggest, however, that the liquid jet diameter is not the critical length scale in the breakup process. As it will be shown in $\S 4$, the critical length scale is rather the primary instability wavelength, $\lambda_{1}$. Recalling that this wavelength is proportional to the gas boundary-layer vorticity thickness, $\delta_{g}$, we 


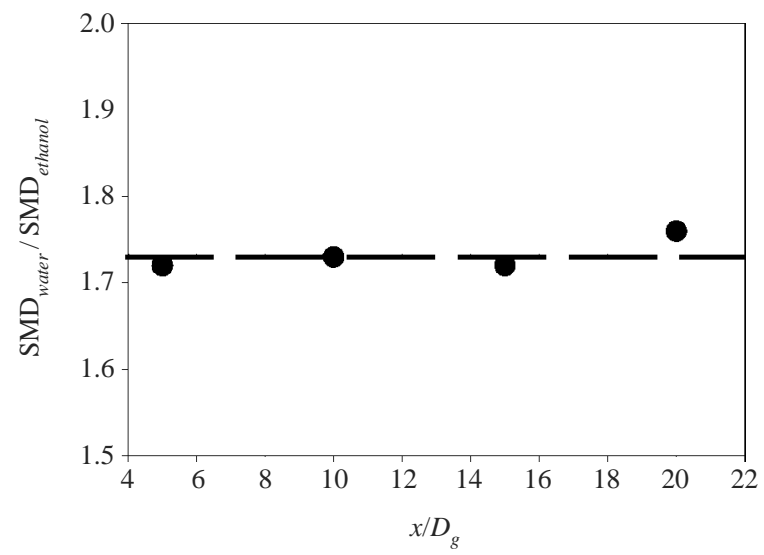

FIGURE 15. SMD ratio of water to ethanol as a function of $x / D_{g}, D_{l}=0.32 \mathrm{~mm}$,

$$
U_{l}=16 \mathrm{~m} \mathrm{~s}^{-1}, U_{g}=165 \mathrm{~m} \mathrm{~s}^{-1} \text {. }
$$

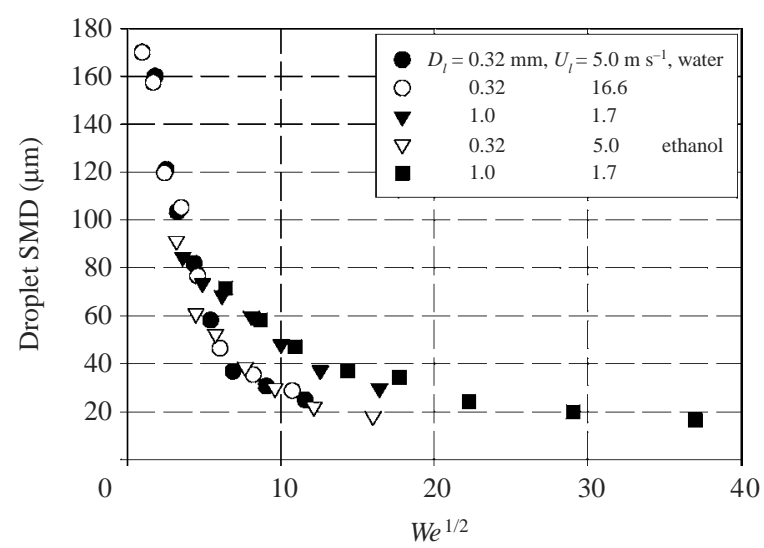

Figure 16. Droplet SMD as a function of $W e^{1 / 2}$.

note that the measurements of figure 12 support this scaling; these results indicate an appreciable dependence of the droplet diameter on the gas boundary-layer thickness, which differs by a non-trivial factor between the two nozzles considered. Accordingly, a new Weber number may be defined based on the length scale $\lambda_{1}$, and is written

$$
W e_{\lambda_{1}}=\frac{\rho_{g}\left(U_{g}-U_{l}\right)^{2} \lambda_{1}}{\sigma} .
$$

An expression for the primary instability wavelength, $\lambda_{1}$, is presented in the following section, and the data of figure 16 are subsequently re-plotted as a function of $W e_{\lambda_{1}}$ to substantiate this scaling argument.

\section{Initial liquid-jet breakup mechanism}

The initial breakup of a low-momentum liquid jet in the presence of a highmomentum co-flowing gas stream has been extensively investigated in previous studies as outlined in $\S 1$. Despite the sizeable amount of research which has been devoted to this two-phase flow scenario, fundamental questions still remain with respect to the physical mechanisms responsible for the initial breakup of the liquid jet. Visual 


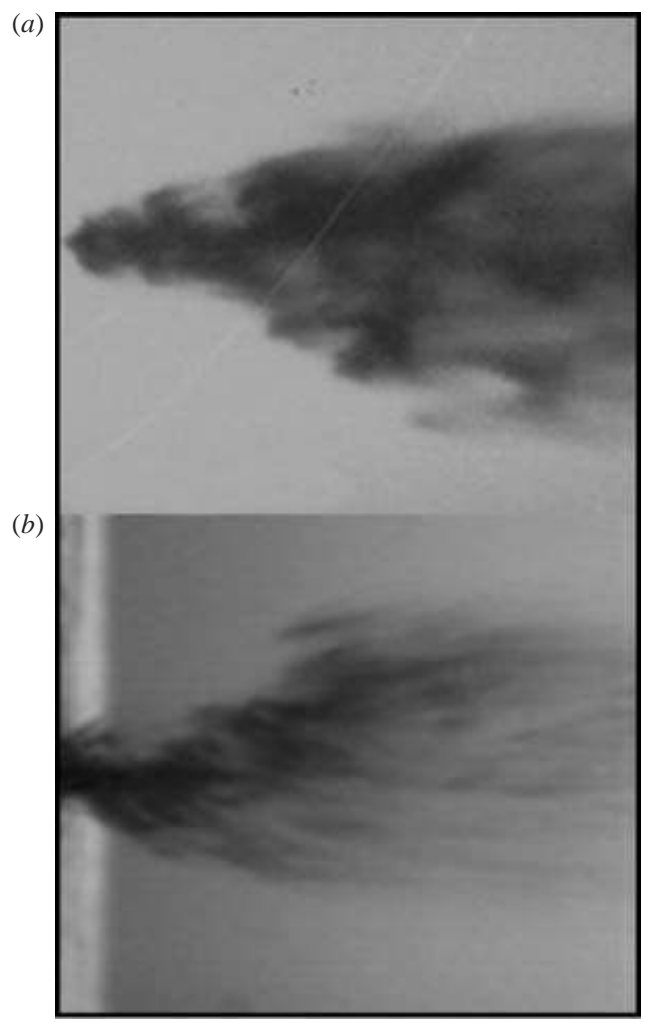

FIGURE 17. Instantaneous flow image comparison of the catastrophic breakup of $(a)$ a $2.5 \mathrm{~mm}$ water droplet (Joseph et al. 1999) and (b) a $1.0 \mathrm{~mm}$ water jet at high Weber number.

observations of the near-nozzle region of the coaxial liquid-gas jet flow in the present experiments (see the images of §3.2) have revealed a mode of liquid jet breakup which appears to share common features with the disintegration of liquid drops in high-speed gas streams. In particular, waves developed at the liquid jet surface by the primary shear instability in the coaxial jet are drawn out into tongues by the surrounding gas stream, and these tongues are subsequently destabilized in a manner which bears a striking resemblance to the accelerative destabilization of drops. This resemblance is illustrated in figure 17 , which compares an image of coaxial jet breakup for the $1.0 \mathrm{~mm}$ diameter liquid jet $(W e=437)$ in the current study to the breakup of a $2.5 \mathrm{~mm}$ water drop $\left(W e=11.7 \times 10^{3}\right)$ in a high-speed air stream (Joseph et al. 1999). These breakup images share several common features, including a similar strippingtype breakup process, the atomization of liquid from distinct sections of destabilized surfaces, and extensive mist formation. It should be noted that the scale by which drop breakup Weber number and jet breakup Weber number should be compared is the wave-crest thickness in the case of the jet, and the flattened drop thickness in the case of drop breakup.

The geometric resemblance of these two flow scenarios was discussed in $\S 1$, and was illustrated in figure 2. Droplet formation appears to occur in both cases through the development of a secondary instability which manifests itself along the surface of the liquid tongues or the drop surface. Droplets appear in images to be 'stripped' from the liquid surface, and a visible liquid mist is often observed to emanate from the wave crests and drop equators in these flows. The amplification of this secondary 
instability may also allow the surface waves to completely penetrate the drop or liquid tongues, forming droplets and liquid fragments which are subject to further breakup by the same mechanisms. This process is referred to as catastrophic breakup and has been documented by Pilch \& Erdman (1987) and others for drop Weber numbers greater than 350. Several conflicting physical explanations currently exist in the literature for the disintegration process in high-speed drop breakup, including arguments involving secondary instability mechanisms such as boundary-layer stripping, Kelvin-Helmholtz instability, Faraday instability, and the Rayleigh-Taylor mechanism proposed here. These breakup mechanisms are briefly reviewed in the following subsection, and are scrutinized in relation to the present experimental results and observations. It is argued that the most plausible initial breakup mechanism, at least in the current coaxial jet experiments, is the secondary, accelerative destabilization of tongues of liquid drawn out of the liquid jet surface by the primary instability; this accelerative destabilization mechanism being the well-known Rayleigh-Taylor instability which develops when a liquid surface is accelerated in a direction perpendicular to its plane. Inconsistencies of other physical explanations and models are pointed out, and compelling experimental evidence from the present study is provided to support the proposed breakup mechanism. A phenomenological breakup model based on the Rayleigh-Taylor instability mechanism is developed in $\S 4.2$, and in $\S 4.3$, comparisons are made between this model and the current experimental data. Existing breakup models are subsequently discussed in the context of these comparisons in $\S 4.4$.

\subsection{Review of existing models for liquid drop breakup in high-speed gas streams}

Three basic regimes of breakup are encountered by liquid drops in high-speed gas streams. These include bag breakup, shear breakup and catastrophic breakup. Transitions between these regimes are typically defined by the value of the Weber number, defined with the initial drop diameter. Bag breakup is encountered in the range $12<W e<80$, and is analogous to membrane breakup in liquid jets, which occurs at approximately the same Weber number, provided $D_{l} \sim \lambda_{1}$. Shear breakup occurs for $W e>80$, and catastrophic breakup, as already pointed out, generally occurs for $W e>350$. For $O h \ll 1$, which is the case in the current experiments, significant drop distortion and oscillations have been observed at We 1 (Liu \& Reitz 1997). Drop distortion is an important initial stage of drop destabilization which is common to all breakup regimes, and plays a key role in the comparison of drop breakup to ligament breakup in coaxial liquid-gas jets.

The cases of most practical value to liquid jet atomization by a high-speed gas stream are those with large Weber numbers where the general concept of shear breakup is valid. Two common theories currently exist for droplet production in the shear breakup regime. The first theory attributes droplet production to the stripping of a liquid boundary layer off the drop near the equator. This liquid boundary layer is assumed to be formed and set in motion by the shear of the gas flow. The second theory attributes droplet production to the breaking of surface waves which form on the windward surface of the drop (Engel 1958).

Hsiang \& Faeth (1992) proposed a boundary-layer stripping mechanism of droplet formation which involves the flattening of the drop by the gas flow into a thin disk, followed by the deflection of the disk periphery in the downstream direction, and the formation of a liquid boundary layer which is stripped from the drop. The thickness of the boundary layer ultimately determines the primary droplet size. Assuming a laminar boundary layer, and that the droplets produced are proportional in size to the boundary-layer thickness at the point of separation, Hsiang \& Faeth found a 
correlating expression for the SMD as, $\mathrm{SMD} / d_{0}=C_{s}\left(\rho_{l} / \rho_{g}\right)^{1 / 4} R e_{l}^{-1 / 2}$, where $C_{s}$ is an empirical constant, $d_{0}$ is the diameter of the drop, and $R e_{l}$ is the liquid Reynolds number based on $d_{0}$ and the gas stream velocity.

Liu \& Reitz (1997) have commented on the fact that the boundary-layer stripping model of Hsiang \& Faeth assumes that viscous shear forces are dominant, and that the drop breakup process thus scales with the Reynolds number. Their results do not support this (nor do our experiments), and they have suggested that the breakup process rather obviously scales with the Weber number (see figures 16 and 29 herein for further evidence of this scaling). A two-stage breakup mechanism has been proposed by Liu \& Reitz for the shear breakup regime, which would apply even to inviscid flows. The first stage involves the distortion of the drop by the air flowing around it, and the second stage involves the production of droplets at the equator owing to a gas-assisted liquid stretching process, which eventually gives way to capillary wave pinching. Extension of the drop by the suction stress at the equator leads to a continual thinning of the drop edges which then tend to be bent and drawn out into thin sheets in the direction of the airflow owing to their low inertia. Droplet formation is proposed to originate from these sheets or filaments through Rayleigh breakup. Villermaux (1998) has proposed a breakup model for primary atomization in coaxial jets based upon a mechanism involving the production of azimuthal waves formed along liquid sheets that ultimately result in the production of ligaments that break up by capillary pinching. This model yields an expression for the primary droplet radius, $R$, of $R / \delta \propto W e_{\delta}^{-1 / 5}\left(\rho_{l} / \rho_{g}\right)^{2 / 5}$, where $\delta$ is the thickness of the gas stream velocity profile.

The second common theory for shear breakup and catastrophic breakup of drops in high-speed gas streams involves the formation of waves on the windward surfaces of drops. Engel (1958) endeavoured to determine the formation mechanism of the mist that he observed in his images to emanate from drops as they were accelerated by the high-speed gas. Surface corrugations on the windward faces of the drops are very clear in the images of his experiments. Pilch \& Erdman (1987) suggested that this generation of mist in high-Weber-number flows occurs through the stripping of wave crests. Engel concluded that the production of mist from liquid drops in high-speed gas flows was probably due to contributions from a number of mechanisms, including both wave-crest breaking and boundary-layer stripping, but that it seemed unlikely that one breakup mechanism would operate to the complete exclusion of all others.

Proposed origins of surface waves in high-speed drop breakup include both the Kelvin-Helmholtz mechanism and the Rayleigh-Taylor instability. Pilch \& Erdman (1987) proposed two droplet-generation processes which stem from the formation of surface waves in high-speed drop breakup. The first involves the stripping or erosion of the wave crests by the high-speed gas stream, and the second involves the amplification of the surface instability to a point where it eventually penetrates the drop entirely. The size of droplets formed through these wave destabilization mechanisms is then reasonably expected to be proportional to the most unstable wavelength of the instability.

Joseph et al. (1999), on the other hand, proposed that drop breakup at high Weber numbers, is controlled at early times by the Rayleigh-Taylor instability, which was introduced in $\S 1$. They have supported this claim with a thorough comparison between theory and experiments, which involved measurements of droplet accelerations and wavelengths of the Rayleigh-Taylor waves in the images of their high-speed films. Their experimental observations were found to share many common features with the breakup regime classifications of Pilch \& Erdman (1987) and Hsiang \& Faeth 
(1992); the authors propose that the surface corrugations observed in the cartoons of Pilch \& Erdman for $W e>350$ are actually Rayleigh-Taylor waves. Joseph et al. (1999) also noted that the waves formed on the windward faces of unstable drops are propagated toward the equator by the shear flow of gas coming from the stagnation point. They recognized the possibility that this shear flow could be subject to the Kelvin-Helmholtz instability, but argued that it should not play a dominant role or interact significantly with the Rayleigh-Taylor instability. A comparison of the growth rates of these two instability mechanisms is made in $\S 4.4$ of this article, and supports this argument.

The most plausible mechanism of initial breakup in the current experiments appears to be associated with the Rayleigh-Taylor instability, and follows the arguments of Joseph et al. (1999). In addition to the observed similarities between the breakup process in the current study and that of the study of Joseph et al., the Rayleigh-Taylor breakup model proposed in the following section is further supported by the clearly observed dependence of the measured droplet SMD on surface tension. The idea of the existence of such a Rayleigh-Taylor instability process finds roots in the observation of pronounced helical movements of the liquid jet just downstream of the injector (see for instance figures 5 and 9) which suddenly expose sections of the liquid jet to the high-speed gas stream. It was initially conjectured that the characteristic length scale which determines the mass to be accelerated should be the liquid jet diameter; however, observations indicated that this is not the case, and that the liquid mass which is accelerated is rather determined by the wavelength of the primary instability. Boundary-layer stripping models and the high-Weber number limit of the Kelvin-Helmholtz instability for a finite shear-layer thickness do not predict any dependence on surface tension, while the pure Kelvin-Helmholtz mechanism predicts $\mathrm{SMD} \propto \sigma$. The scaling dependence of the mean droplet size on surface tension indicated by the present experimental results appears to be $\mathrm{SMD} \propto \sigma^{1 / 2}$, which is precisely the dependence predicted by the Rayleigh-Taylor instability.

\subsection{Phenomenological initial breakup model}

In this section, we systematically develop a phenomenological model for the initial breakup of a liquid jet by a high-speed coaxial gas stream. Figure 18 shows a schematic of the experimentally observed initial breakup process in the present study. Waves of length $\lambda_{1}$ are formed at the gas-liquid interface by the primary shear instability. The action of the high-speed gas on this wave structure acts to draw the waves out into tongues of a characteristic thickness $b$. Figure 19 shows the envisaged streamlines of both the gas and liquid flows. As the liquid jet is rapidly accelerated by the highspeed gas flow, its diameter is accordingly reduced, and the stagnation point balance between the liquid and gas flows is presumed to be offset as shown in the figure. This process removes the support of the liquid tongue by the bulk liquid, and the liquid tongue becomes vulnerable to the gas flow as it is further drawn out of the liquid surface. The surfaces of these tongues are subjected to strong accelerations by the surrounding gas flow, which destabilizes them via the well-known Rayleigh-Taylor instability, forming corrugations of a characteristic wavelength $\lambda_{R T}$. These RayleighTaylor waves become amplified, and the integrity of the liquid tongue is eventually broken, as droplets are formed with a typical size $d$, where $d \propto \lambda_{R T}$. This combined process involving the primary shear instability and the liquid surface destabilization by Rayleigh-Taylor waves is shown clearly in the high-speed images of figures 20 and 21 (i.e. $\lambda_{1}$ in figure 20 , and $\lambda_{R T}$ in figure 21). 

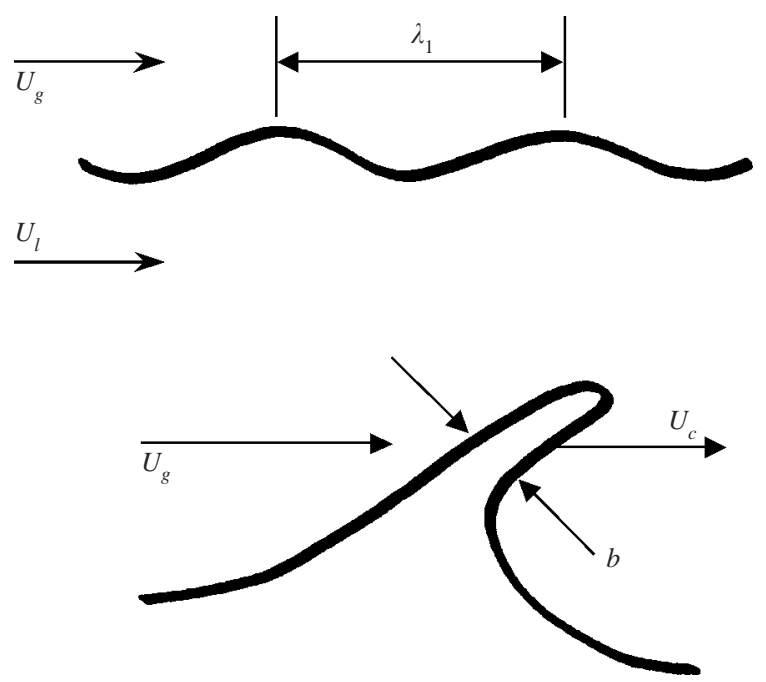

FIGURE 18. Schematic of the development of the liquid jet breakup process.

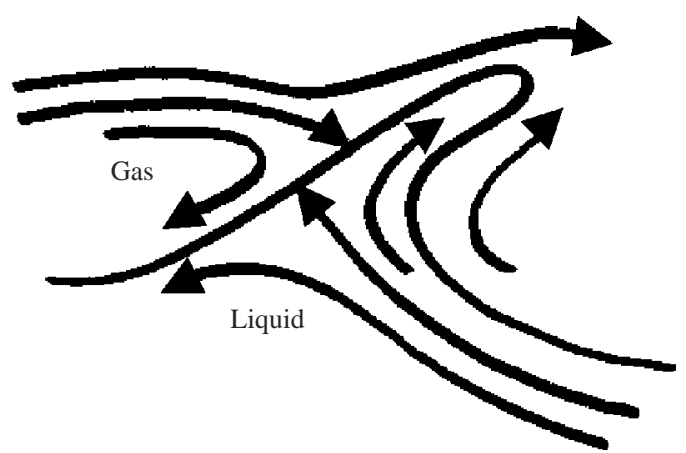

FIGURE 19. Sketch of the gas and liquid streamlines in the liquid tongue formation process.

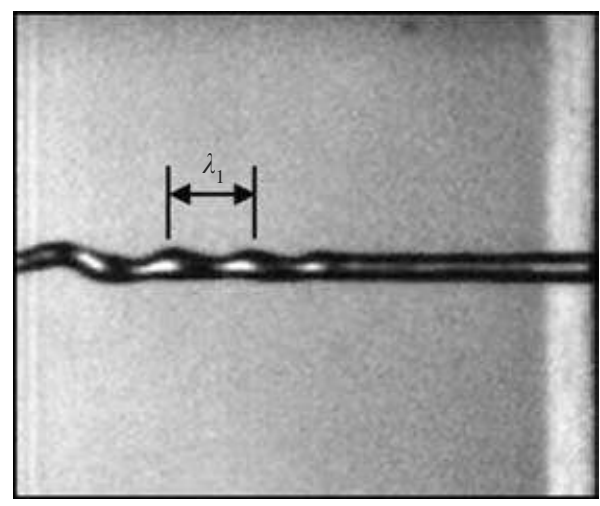

FIGURE 20. Instantaneous flow image illustrating the primary shear instability with wavelength $\lambda_{I}, D_{l}=0.32 \mathrm{~mm}, U_{g}=40 \mathrm{~m} \mathrm{~s}^{-1}$.

To develop the breakup model, we begin by considering the surface between a gas of constant density $\rho_{g}$ and a liquid of constant density $\rho_{l}$; this surface corresponds to the liquid tongue surface and the surrounding gas flow. The flow field is subjected to a 


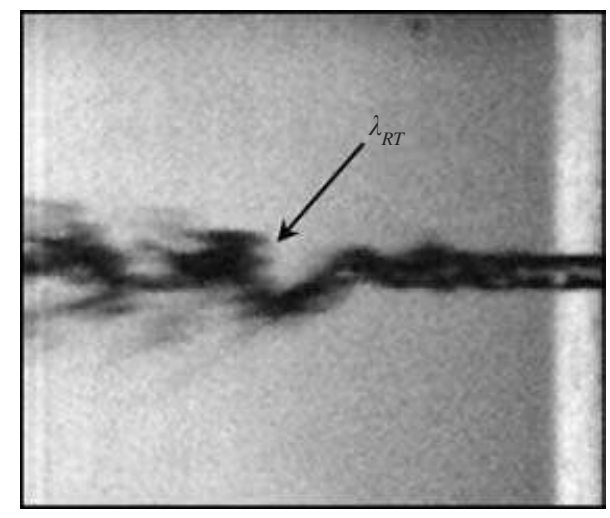

FIGURE 21. Instantaneous flow image illustrating the development of Rayleigh-Taylor waves on the accelerating liquid jet surface, $D_{l}=0.32 \mathrm{~mm}, U_{g}=100 \mathrm{~m} \mathrm{~s}^{-1}$.

constant acceleration, $a$, perpendicular to the surface of separation, and acceleration due to gravity is neglected (high-Froude number limit). The classic linear stability analysis of this problem, including the effects of surface tension (Chandrasekhar 1961), yields the familiar Rayleigh-Taylor dispersion relation

$$
\omega^{2}=\frac{k\left(\left(\rho_{l}-\rho_{g}\right) a-k^{2} \sigma\right)}{\rho_{l}+\rho_{g}},
$$

where $\omega$ is the temporal growth rate, and $k$ is the wavenumber. The critical wavelength corresponding to the border of instability/stability is given by $\omega=0$ in (4.1), which, for $\rho_{l} \gg \rho_{g}$, yields

$$
\lambda_{c}=2 \pi \sqrt{\frac{\sigma}{\rho_{l} a}} .
$$

Thus, all waves of size $\lambda>\lambda_{c}$ are unstable and will grow in time. The wavelength of most relevance to the liquid jet breakup process, however, is the most unstable wavelength, or the wavelength with the maximal growth rate. Calculation of this wavelength, which we have identified as $\lambda_{R T}$, by differentiation of (4.1), yields

$$
\lambda_{R T}=\sqrt{3} \lambda_{c}=2 \pi \sqrt{\frac{3 \sigma}{\rho_{l} a}} .
$$

We now proceed to estimate the acceleration of the wave tongues. To estimate this acceleration term in (4.2) and (4.3), we consider the acceleration of a volume of the liquid jet, $\chi$, in the presence of the co-flowing gas stream, expressed as

$$
a=\frac{\mathrm{d} V_{l}}{\mathrm{~d} t}=\frac{F}{m_{l}}=\frac{F}{\rho_{l} \chi},
$$

where $V_{l}$ is the velocity of the liquid mass, and $m_{l}$ is the mass of the liquid segment. The force, $F$, consists of the drag and is expressed in the usual way as

$$
F=F_{D}=C_{D} \frac{1}{2} \rho_{g}\left(U_{g}-U_{c}\right)^{2} A_{e},
$$

where $C_{D}$ is the drag coefficient, $U_{g}$ is the bulk gas exit velocity, $U_{c}$ is the convection velocity of the liquid surface waves, and $A_{e}$ is the exposed surface area of the liquid volume. The convection velocity, $U_{c}$, is given by the familiar expression for mixing 
layers with streams of differing densities (Dimotakis 1986),

$$
U_{c}=\frac{\sqrt{\rho_{l}} U_{l}+\sqrt{\rho_{g}} U_{g}}{\sqrt{\rho_{l}}+\sqrt{\rho_{g}}} .
$$

Equation (4.6) has been shown to accurately predict measured convection velocities in two-phase coaxial jets (Raynal 1997). Equation (4.6) may be simplified for the case of interest here, wherein $\rho_{l} \gg \rho_{g}$, as

$$
U_{c}=U_{l}+\sqrt{\frac{\rho_{g}}{\rho_{l}}} U_{g} .
$$

Substituting (4.5) and (4.7) into (4.4) yields the acceleration

$$
a=\frac{C_{D} \rho_{g}\left(U_{g}\left(1+\sqrt{\rho_{g} / \rho_{l}}\right)-U_{l}\right)^{2} A_{e}}{2 \rho_{l} \chi},
$$

which yet requires the determination of $C_{D}, A_{e}$ and $\chi$ to make it appropriate for use in (4.3) for the most-amplified Rayleigh-Taylor instability wavelength, $\lambda_{R T}$.

The drag coefficient, $C_{D}$, can be reasonably estimated from experimental data for fragmenting drops under comparable flow conditions, assuming that the tongues of liquid in the present case of a liquid jet are subjected to similar influences. These influences include liquid mass losses and surface deformation, which are typically accounted for through an average drag coefficient obtained by performing a curve fit to drop displacement data. Pilch \& Erdman (1987) have summarized the drag coefficients for fragmenting drops obtained by a number of authors, with $C_{D}$ ranging from 1.5 to 2.9 for a large range of Weber numbers. For the range of Weber numbers considered in the current experiments, $C_{D}$ ranges from approximately 1.5 to 2.7 , so an average value of $C_{D}=2$ was chosen for this analysis. As shown in figure 18, the segments of the liquid jet which are accelerated by the high-speed gas stream consist of tongues of liquid of characteristic thickness $b$. The volume (per unit width) of these elements is given by $\chi=b A_{e}$, which upon substitution into (4.8) along with $C_{D}=2$ yields

$$
a=\frac{\rho_{g}\left(U_{g}\left(1+\sqrt{\rho_{g} / \rho_{l}}\right)-U_{l}\right)^{2}}{\rho_{l} b} .
$$

The thickness $b$ of the liquid tongues must sensibly be related to another length scale in the flow. This thickness appears to be independent of the liquid jet diameter, which is not surprising considering that these tongues of liquid are formed through the aerodynamic enhancement of the primary instability, which has a wavelength, $\lambda_{1}$, prescribed by the coaxial gas stream. Therefore, we take $b \propto \lambda_{1}$, or $b=\alpha \lambda_{1}$, and seek to empirically determine the proportionality constant $\alpha$. The present experimental observations indicate that $\alpha \approx 1 / 10$ is reasonable. Upon substitution of $b=\lambda_{1} / 10$ into (4.9), we obtain

$$
a \approx \frac{10 \rho_{g}\left(U_{g}\left(1+\sqrt{\rho_{g} / \rho_{l}}\right)-U_{l}\right)^{2}}{\rho_{l} \lambda_{1}} .
$$

Equation (4.10) requires knowledge of the primary instability wavelength, $\lambda_{1}$, in order for it to be used in (4.3) to estimate the sizes of fragments produced through this breakup mechanism. Therefore, we proceed now to develop an expression for this wavelength.

The primary instability at the gas-liquid interface which determines the wavelength $\lambda_{1}$ is the well-known Kelvin-Helmholtz instability mechanism that occurs at the 
interface of finite-vorticity-thickness, parallel flowing streams of fluid in relative motion. Analyses of this instability in both planar and coaxial liquid-gas flows in the high-Weber number limit (i.e. neglecting surface tension) were carried out by Raynal (1997). Raynal showed that a simplified inviscid linear stability analysis in planar coordinates, which considered a boundary layer in the gas stream only, yields accurate results for primary wavelengths measured experimentally with air and water. As discussed in $\S 1$, the results of Raynal indicated that the primary instability wavelength is proportional to the vorticity-layer thickness in the gas stream, as well as the density ratio in the form

$$
\lambda_{1}=C\left(\frac{\rho_{l}}{\rho_{g}}\right)^{1 / 2} \delta_{g} .
$$

(Note that this wavelength is much larger than the classical Kelvin-Helmholtz instability wavelength of a discontinuous interface, $\lambda_{K H}=2 \pi \sigma\left(\rho_{l}+\rho_{g}\right) / \rho_{l} \rho_{g}\left(U_{g}-U_{l}\right)^{2}$. The amplification rate is however the same, and is given by $\sigma=k \sqrt{\rho_{g} \rho_{l}}\left(U_{g}-U_{l}\right) /$ $\left(\rho_{l}+\rho_{g}\right)$, where $k \simeq\left(\rho_{g} / \rho_{l}\right)^{1 / 2} \delta_{g}{ }^{-1} \ll k_{K H}$.) The vorticity-layer thickness of the gas boundary layer, $\delta_{g}$, and the coefficient $C$ are dependent on the particular gas nozzle geometry considered. In planar liquid/gas shear layers, experiments suggest $C \simeq 4$, which is close to the theoretical value (Raynal 1997). In the coaxial, axisymmetric jet configuration of interest, $C \simeq 1.2$ (Marmottant 2001). For simplicity, we have limited our consideration here to the convergent gas nozzle geometry described in $\S 2.1$, which possesses thin laminar boundary layers at the nozzle exit plane. Thus, in general, the boundary-layer thickness (proportional to the vorticity thickness, $\delta_{g}$ ) scales as $\left(v_{g} / U_{g}\right)^{1 / 2}$, and we write

$$
\lambda_{1}=\gamma\left(\frac{\rho_{l}}{\rho_{g}}\right)^{1 / 2}\left(\frac{\nu_{g}}{U_{g}}\right)^{1 / 2},
$$

where $\gamma$ is a proportionality factor that accounts for the specifics of the particular nozzle geometry, including $C$. Substituting (4.12) into (4.10), we arrive at our final expression for the liquid acceleration term,

$$
a=\frac{10}{\gamma} \frac{1}{v_{g}^{1 / 2}}\left(\frac{\rho_{g}}{\rho_{l}}\right)^{3 / 2}\left[U_{g}\left(1+\sqrt{\frac{\rho_{g}}{\rho_{l}}}\right)-U_{l}\right]^{2} U_{g}^{1 / 2} .
$$

Upon substitution of (4.13) into (4.3), we obtain the desired expression for the most amplified Rayleigh-Taylor instability wavelength, as

$$
\lambda_{R T} \approx \frac{\kappa \xi}{\left[U_{g}\left(1+\sqrt{\rho_{g} / \rho_{l}}\right)-U_{l}\right] U_{g}{ }^{1 / 4}},
$$

where $\kappa$ is a constant factor (for a given nozzle geometry),

$$
\kappa=2 \pi\left(\frac{3 \gamma}{10}\right)^{1 / 2}
$$

and $\xi$ is a fluid properties factor,

$$
\xi=\sigma^{1 / 2}\left(\frac{v_{g} \rho_{l}}{\rho_{g}^{3}}\right)^{1 / 4} .
$$

The diameter of the droplets is assumed to be proportional to this secondary instability wavelength, $\lambda_{R T}$, an assumption which is supported by the experiments of Marmottant (2001). In the following section, we compute $\kappa$ and $\xi$ for the present experiments and compare predicted wavelengths from (4.14) to our experimental results. 


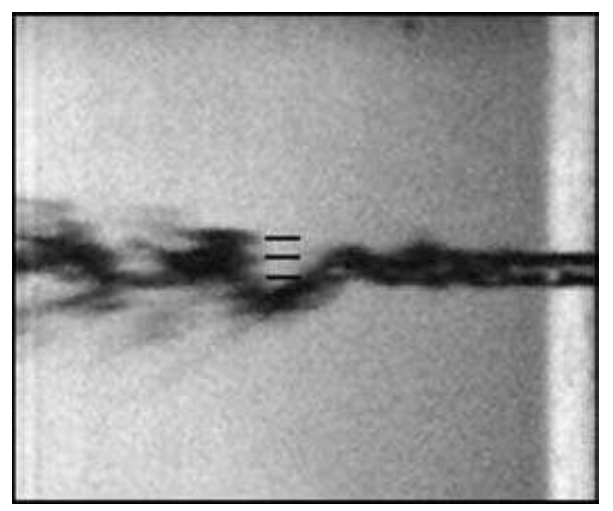

FIGURE 22. Instantaneous flow image with identified Rayleigh-Taylor wavelengths, $D_{l}=0.32 \mathrm{~mm}, W e=37, \lambda_{\text {measured }}=200 \mu \mathrm{m}$.

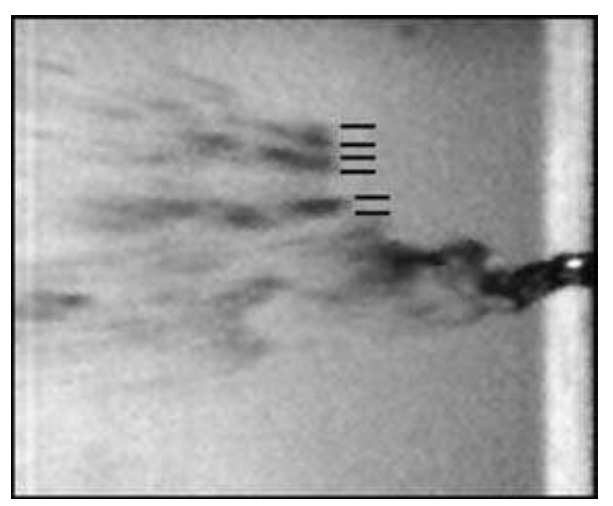

FIGURE 23. Instantaneous flow image with identified Rayleigh-Taylor wavelengths, $D_{l}=0.32 \mathrm{~mm}, W e=47, \lambda_{\text {measured }}=185 \mu \mathrm{m}$.

\subsection{Comparison of the model to experimental results}

The model developed in $\S 4.2$ was evaluated by comparison to a subset of the experimental results presented in $\S 3.2$. Figures 22-26 comprise several characteristic high-speed video images which are representative of the initial liquid-jet breakup process over a range of Weber numbers. In a manner similar to that of Joseph et al. (1999), predicted Rayleigh-Taylor wavelengths from (4.14) were compared to measured values from these high-speed video images. In each figure, tick marks have been placed on the image to indicate the most evident wave spacing, which was measured using computer software tools. To estimate the proportionality constant $\gamma$ in (4.12), we have used experimental measurements of $\lambda_{1}$ from images such as that shown in figure 20. From this analysis, we have estimated $\gamma \approx 0.055 \mathrm{~m}^{1 / 2}$, which yields $\kappa=0.807 \mathrm{~m}^{1 / 4}$. The value $\gamma \approx 0.055 \mathrm{~m}^{1 / 2}$ gives $\delta_{g} \simeq 0.6 e / \sqrt{R e}$, where $e=$ $\left(D_{g}-D_{l, o}\right) / 2$ and $R e=e U_{g} / \nu_{g}$, when $C \simeq 1.2$ in (4.11). To calculate the fluid properties factor, $\xi$, we refer to the values in table 1 , which yield $\xi=0.082 \mathrm{~m}^{2} \mathrm{~s}^{-5 / 4}$. Table 2 summarizes the comparisons for the five image subset. The predicted wavelengths from the phenomenological model of (4.14) show very good agreement with the measured wavelengths from the images; the agreement was on average within $14 \%$. The uncertainty in the measurements of the wavelengths in the images of figures $22-26$ has not been quantified. Under the present conditions of large gas velocity, precise 


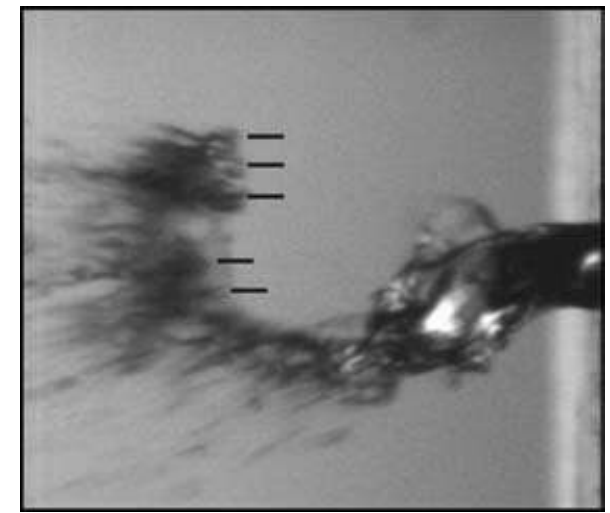

FIGURE 24. Instantaneous flow image with identified Rayleigh-Taylor wavelengths, $D_{l}=1.0 \mathrm{~mm}, W e=74, \lambda_{\text {measured }}=310 \mu \mathrm{m}$.

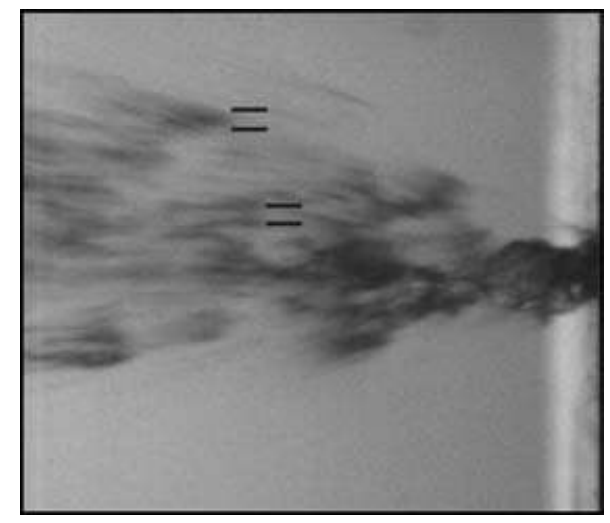

FIGURE 25. Instantaneous flow image with identified Rayleigh-Taylor wavelengths, $D_{l}=1.0 \mathrm{~mm}, W e=158, \lambda_{\text {measured }}=235 \mu \mathrm{m}$.

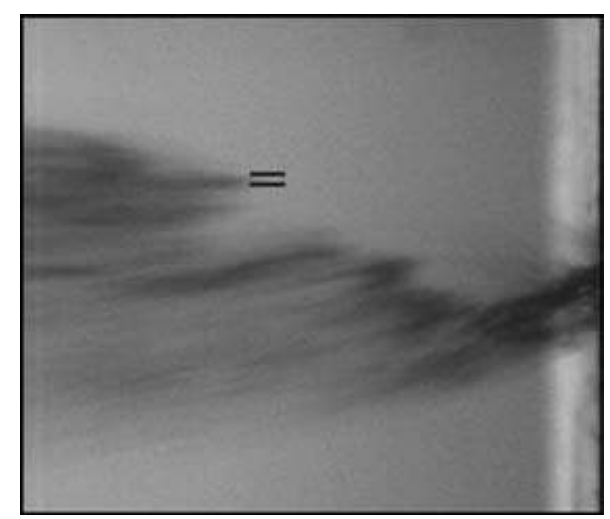

FiguRE 26. Instantaneous flow image with identified Rayleigh-Taylor wavelengths, $D_{l}=1.0 \mathrm{~mm}, W e=437, \lambda_{\text {measured }}=95 \mu \mathrm{m}$.

measurements on images are difficult, but nevertheless give an indication that the order of magnitude is correct. We did not encounter any realizations of the breakup process which were in gross disagreement with our model predictions; all of our measurements 


$\begin{array}{crccccc}\text { Figure } & W e & U_{g}\left(\mathrm{~m} \mathrm{~s}^{-1}\right) & U_{l}\left(\mathrm{~m} \mathrm{~s}^{-1}\right) & \lambda_{1}(\mathrm{~mm}) & \lambda_{R T} \text { Predicted }(\mu \mathrm{m}) & \lambda_{R T} \text { Measured }(\mu \mathrm{m}) \\ 22 & 37 & 100 & 16.5 & 0.484 & 242 & 200 \\ 23 & 47 & 100 & 4.9 & 0.484 & 213 & 185 \\ 24 & 74 & 69 & 1.7 & 0.582 & 331 & 310 \\ 25 & 158 & 100 & 1.7 & 0.484 & 207 & 235 \\ 26 & 437 & 165 & 1.7 & 0.377 & 110 & 95\end{array}$

TABLE 2. Comparison of model predictions with experimental data.

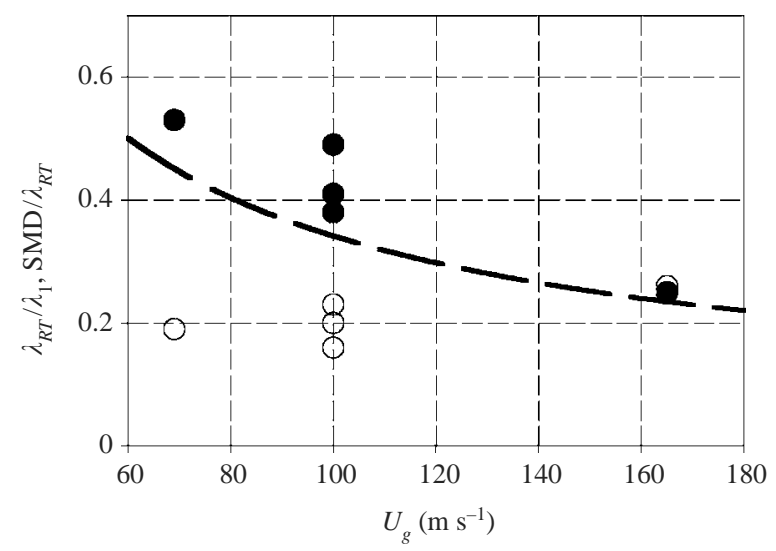

FIGURE 27. $\bullet, \lambda_{R T} / \lambda_{I}$ and $\bigcirc, \mathrm{SMD} / \lambda_{R T}$ as a function of $U_{g}$, water, $U_{l}=1.7-16.6 \mathrm{~m} \mathrm{~s}^{-1}$, $D_{l}=0.32,1.0 \mathrm{~mm}$. The line shows $\lambda_{R T} / \lambda_{1}$ predicted.

are consistent with the proposed Rayleigh-Taylor instability mechanism. Additional direct support in favour of the Rayleigh-Taylor instability is given by the droplet-size measurements. It should be noted that as the Rayleigh-Taylor wavelength approaches the resolution of our images, the uncertainties in the wavelength measurements may become large.

Figure 27 contains plots of the ratios of $\lambda_{R T} / \lambda_{1}$ as well as $\mathrm{SMD} / \lambda_{R T}$ for the flow cases given in table 2 . The experimentally measured secondary-to-primary wavelength ratio $\left(\lambda_{R T} / \lambda_{1}\right)$ is observed to show good agreement with the decay predicted by the division of (4.14) by (4.12) (i.e. $\lambda_{R T} / \lambda_{1} \propto U_{g}^{-3 / 4}$ ), shown as a dashed line in figure 27 . Figure 27 also suggests that (on average) $\mathrm{SMD} \approx \lambda_{R T} / 5$, which permits the extension of (4.14) to provide a practical expression for the Sauter mean diameter

$$
\mathrm{SMD} \approx \frac{0.68 \gamma^{1 / 2}\left(\rho_{l} \nu_{g}\right)^{1 / 4} \sigma^{1 / 2}}{\rho_{g}^{3 / 4}\left[U_{g}\left(1+\sqrt{\rho_{g} / \rho_{l}}\right)-U_{l}\right] U_{g}^{1 / 4}} .
$$

The dependence on surface tension predicted by the current phenomenological model $\left(d \propto \sigma^{1 / 2}\right)$ is supported by the measurements of $\S 3$, and has been viewed here as a footprint of the Rayleigh-Taylor instability in the primary breakup process. The gas velocity dependence of this model is also significant, particularly in light of the historical value which has been placed on obtaining correlations of the form, $\mathrm{SMD} \propto U_{g}{ }^{-n}$, as discussed in $\S 1$. Equation (4.17) predicts a mean droplet diameter dependence of $U_{g}^{-5 / 4}$, or $n=1.25$. Figure 28 shows two plots at $x / D_{g}=15$ of droplet SMD as a function of bulk gas exit velocity, $U_{g}$, for two sets of flow conditions. Power-law curves have been fit to these data sets to determine the exponent $n$, which 


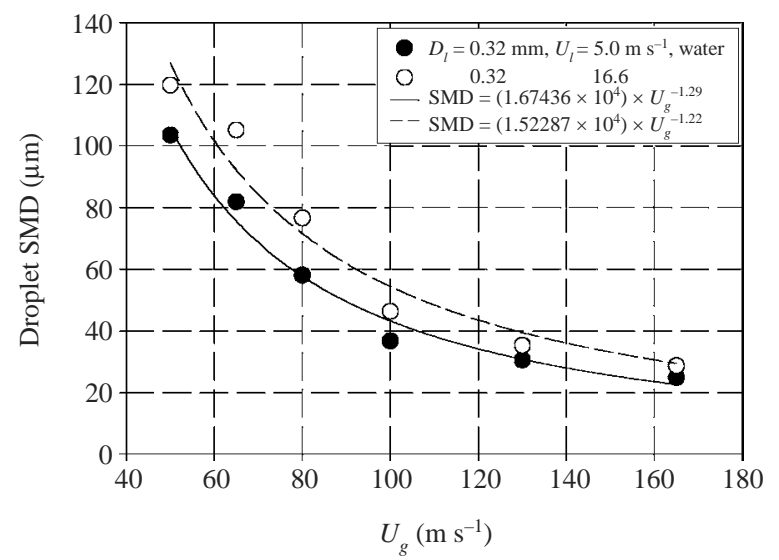

FIGURE 28. Droplet SMD as a function of atomizing gas velocity.

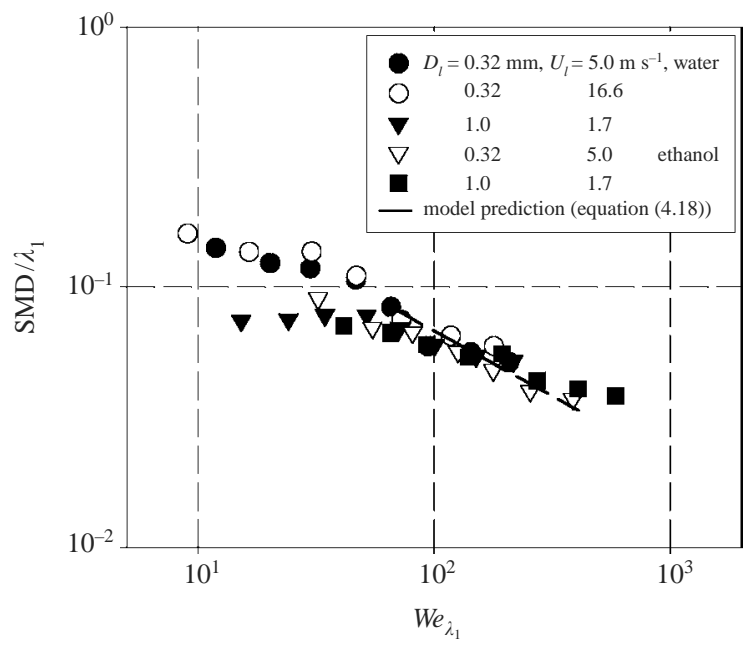

FIGURE 29. Droplet SMD $/ \lambda_{1}$ as a function of $W e_{\lambda_{1}}$.

has been compared to the value of $n=1.25$ predicted by the current phenomenological Rayleigh-Taylor instability model. The best fits to these data sets are observed to yield $n=1.22$ and $n=1.29$, which agree very well with the model prediction of $n=1.25$.

For the present case of interest, where $\rho_{g} \ll \rho_{l}$, (4.17) may be rewritten in terms of $W e_{\lambda_{1}}$, as defined in (3.3), yielding the relation

$$
\frac{\mathrm{SMD}}{\lambda_{1}} \approx \frac{0.68}{W e_{\lambda_{1}}^{1 / 2}} .
$$

Figure 29 contains the droplet-size data of figure 16, scaled by the primary instability wavelength, $\lambda_{1}$, and re-plotted as a function of $W e_{\lambda_{1}}$. The data from the five different experiments, involving both water and ethanol, collapse very well over nearly a decade in $W e_{\lambda_{1}}$ to the power law dependence predicted by (4.18), beginning at $W e_{\lambda_{1}} \approx 60$. This Weber number corresponds to the point at which $\lambda_{R T} / \lambda_{1} \sim 0.5$ (see figure 27 with $U_{g} \sim 65 \mathrm{~m} \mathrm{~s}^{-1}$ ), the point at which roughly two Rayleigh-Taylor waves fit into one primary instability wavelength. The condition $\lambda_{R T}<\lambda_{1}$ is fulfilled for $W e_{\lambda_{1}}>10$; the 
development of multiple waves is reasonably expected to coincide with the dominance of the Rayleigh-Taylor mechanism in the breakup of the liquid tongues.

The impact of secondary breakup mechanisms on the droplet sizes presented in figures 27-29 for comparison with the model has also been considered. Because the liquid jet is of small dimension in the present case, breakup is completed well before the measuring station; the images clearly show this. The measured droplets at $x / D_{g}=15$ are therefore indicative of the primary breakup process. The actual size distribution at this location is, however, affected by relative acceleration and to some extent by collisions. Turbulent secondary breakup is of no importance for the current conditions, as the droplets produced by the primary breakup process are already small. Note also, as discussed in $\S 3.3$, that because the liquid jet experiences large radial excursions from the centreline, the measurements at the centre of the spray are expected to be indicative of the overall breakup process.

The agreement between the SMD data given in figure 28 and the gas-velocity scaling predicted by the current model, combined with the clear scaling on $W e_{\lambda_{1}}$ exhibited by the data of figure 29, provide further support to the claim that the Rayleigh-Taylor instability is dominantly involved in the primary breakup process for coaxial jets under the conditions of the current experimental study.

\subsection{Comparison of the present model and experiments to alternative breakup models}

It is of interest to note the dependencies on both gas velocity and liquid surface tension which are predicted by several other breakup models discussed earlier in this section, and others which have been proposed for primary breakup in two-phase coaxial jets. Boundary-layer stripping models predict that the SMD should scale with $d_{0} R e_{l}^{-1 / 2}$, where $d_{0}$ represents the initial drop size. In the breakup of the current liquid jet, the relevant liquid boundary layer would develop over the length of the primary instability wavelength, $\lambda_{1}$ (i.e. $\lambda_{1}$ replaces $d_{0}$ ), implying that the SMD scales as $\lambda_{1} R e_{\lambda_{1}}^{-1 / 2} \propto$ $U_{g}^{-1 / 2}\left(U_{g}^{1 / 2}\right)^{-1 / 2}=U_{g}^{-3 / 4}$, or $n=0.75$. On the other hand, if the Kelvin-Helmholtz instability was the dominant secondary instability which led to droplet formation, the SMD would scale with the wavelength of Kelvin-Helmholtz corrugations formed on the scale of $\lambda_{1}$. The droplet SMD would thus scale with the gas boundary-layer thickness developed over the length $\lambda_{1}$, which scales as $\left(v_{g} \lambda_{1} / U_{g}\right)^{1 / 2}$. This yields, as in the case of boundary-layer stripping, SMD $\propto U_{g}^{-3 / 4}$. Neither of these models predict any dependence of the mean droplet diameter on surface tension, which is striking in light of the present experimental observations, even for large Weber numbers. The Rayleigh-Taylor, or more precisely, the Faraday instability model proposed by Marmottant (2001), predicts a surface tension dependence of $d \propto \sigma^{1 / 3}$, which is weaker than the dependence suggested by the results of the present study, and moreover predicts $n=1$. It is also noteworthy that the process of stretch-assisted sheet disintegration by capillary instability proposed by Liu \& Reitz (1997) and Villermaux (1998), yields $d \propto U_{g}^{-4 / 5}$.

The success of the present model in predicting experimentally observed instability wavelengths and fragment sizes, as well as the correct surface tension dependence and gas velocity scaling, does not preclude the probability that other breakup mechanisms are simultaneously at work in these flows. Joseph et al. (1999) have pointed out that in their high-speed-drop breakup studies, the main mechanism of breakup appears to be associated with the Rayleigh-Taylor instability, but that other mechanisms of instability can lead to droplet formation, as has been documented by Pilch \& Erdman (1987), and Hsiang \& Faeth (1992) for example. 


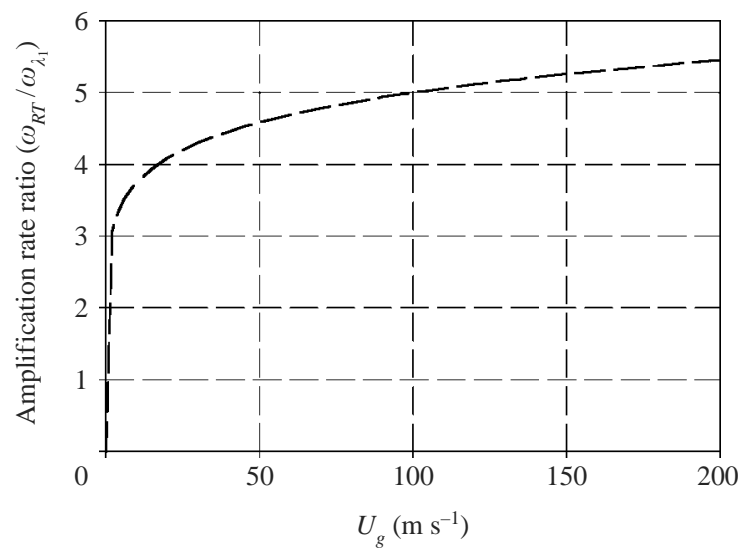

FIGURE 30. Amplification rate ratio as a function of atomizing gas velocity for the Rayleigh-Taylor and Kelvin-Helmholtz instability mechanisms.

The Kelvin-Helmholtz instability is perhaps the most plausible potential cocontributor in the secondary destabilization of primary interfacial waves. The likelihood of this mechanism competing with the Rayleigh-Taylor instability can be assessed through a comparison of the amplification rates of these two instabilities. The maximal growth rate of the Rayleigh-Taylor instability (for $\rho_{l} \gg \rho_{g}$ ) can be calculated from (4.1) with the most unstable wavenumber $k=\sqrt{\rho_{l} a / 3 \sigma}$, as

$$
\omega_{R T}=\left(\frac{2}{3 \sqrt{3}}\right)^{1 / 2}\left(\frac{\rho_{l}}{\sigma}\right)^{1 / 4} a^{3 / 4} .
$$

Substituting (4.10) for the acceleration term in the limit of $U_{g} \gg U_{l}$, we obtain

$$
\omega_{R T}=\left(\frac{2}{3 \sqrt{3}}\right)^{1 / 2}\left(\frac{\rho_{l}}{\sigma}\right)^{1 / 4}\left(\frac{10 \rho_{g} U_{g}^{2}}{\rho_{l} \lambda_{1}}\right)^{3 / 4} .
$$

The amplification rate of the Kelvin-Helmholtz instability, on the other hand, is estimated as $\omega_{K H} \sim U_{c} / \delta_{\lambda_{1}}$, where $\delta_{\lambda_{1}}$ is the fast stream boundary-layer thickness which forms over the extent of $\lambda_{1}$. Therefore, in the limit of $U_{g} \gg U_{l}$, the Kelvin-Helmholtz amplification rate is written

$$
\omega_{K H} \sim \frac{\rho_{g} U_{g}}{\rho_{l}}\left(\frac{U_{g}}{v_{g} \lambda_{1}}\right)^{1 / 2} .
$$

The ratio of (4.20) to (4.21) yields the amplification rate ratio

$$
\frac{\omega_{R T}}{\omega_{K H}} \approx 3.5\left(\frac{\rho_{l}}{\sigma}\right)^{1 / 4}\left(\frac{\rho_{l} v_{g}^{2}}{\rho_{g} \lambda_{1}}\right)^{1 / 4},
$$

and substituting (4.12) for $\lambda_{1}$ into this expression, we obtain the result

$$
\frac{\omega_{R T}}{\omega_{K H}} \approx 3.5\left(\frac{\rho_{l}}{\sigma}\right)^{1 / 4}\left(\frac{\rho_{l}}{\rho_{g}}\right)^{1 / 8} \frac{v_{g}^{3 / 8}}{\gamma^{1 / 4}} U_{g}^{1 / 8} .
$$

The amplification rate ratio expressed by (4.23) is plotted in figure 30 as a function of the atomizing gas velocity, $U_{g}$, over the range of applicability in the present study. Figure 30 illustrates that the growth rate of the Rayleigh-Taylor instability is 
significantly ( $\sim 5$ times) larger than that of the Kelvin-Helmholtz mechanism over the entire range of gas velocities, and furthermore, is a weakly increasing function of $U_{g}$. The amplification and growth of the Kelvin-Helmholtz instability is therefore expected to be overshadowed by the rapid onset of the Rayleigh-Taylor instability on the primary wave structure. This supports the comments put forth by Joseph et al. (1999), which suggested that the Kelvin-Helmholtz instability should not interact significantly with the Rayleigh-Taylor instability in these flows, and serves to solidify the foundation of the model presented here.

\section{Conclusions}

The breakup and atomization of a small-diameter liquid jet by a high-speed gas stream has been investigated experimentally. The observed breakup process is consistent with a mechanism involving the combination of an interfacial and a RayleighTaylor instability of the liquid jet. This instability manifests itself very close to the nozzle exit as amplification of the primary instability causes the liquid jet to make non-trivial lateral excursions from the centreline. This process exposes segments of the liquid jet to large accelerations in a direction perpendicular to their surfaces, making them susceptible to the Rayleigh-Taylor instability. This mechanism ultimately leads to a catastrophic breakup process in which the liquid jet is stripped and reduced to a fine spray, completely within the potential cone of the large-diameter gas jet.

A proposed Rayleigh-Taylor initial breakup model based on the acceleration of tongues of liquid drawn out of the jet surface has been demonstrated to give results which agree very well with experimental mean droplet-size results for the breakup of both water and ethanol jets. The wavelength of the primary gas-liquid interfacial instability was confirmed to be the dominant length scale in determining the thickness of the liquid tongues, which are eventually destabilized by the large accelerations. Primary droplet sizes have been shown to scale well on the most unstable RayleighTaylor wavelength, and the dependence of the droplet diameter on both the atomizing gas velocity and the liquid surface tension has been successfully captured by the proposed phenomenological breakup model.

This research was sponsored by the Office of Naval Research under Grant N0001499-1-0745. We would also like to acknowledge the valuable input of Professor Forman Williams at UC San Diego.

\section{REFERENCES}

BURICK, R. J. 1972 Space storable propellant performance program. Coaxial injectors characterization. NASA CR 120936.

Chandrasekhar, S. 1961 Hydrodynamic and Hydromagnetic Stability. Clarendon.

Chigier, N. \& ReITZ, R. D. 1995 Regimes of jet breakup and breakup mechanisms (physical aspects). In Recent Advances in Spray Combustion: Spray Atomization and Drop Burning Phenomena (ed. K. K. Kuo), vol. 1, pp. 109-135.

Dimotakis, P. E. 1986 Two-dimensional shear layer entrainment. AIAA J. 24, 1791-1796.

Engel, O. G. 1958 Fragmentation of waterdrops in the zone behind an air shock. J. Res Natl. Bur. Stand. 60, 245-280.

FARAgò, Z. \& Chigier, N. 1992 Morphological classification of disintegration of round liquid jets in a coaxial air stream. Atom. Sprays 2, 137-153.

Hardalupas, Y. \& Whitelaw, J. H. 1994 Characteristics of sprays produced by coaxial airblast atomizers. J. Propul. Power 10, 453-460.

von Helmholtz, H. 1868 On discontinuous movements of fluids. Phil. Mag. 36, 337-346. 
Hsiang, L.-P. \& FAeth, G. M. 1992 Near-limit drop deformation and secondary breakup. Intl J. Multiphase Flow. 18, 635-652.

Hwang, S. S., LiU, Z. \& Reitz, R. D. 1996 Breakup mechanisms and drag coefficients of high-speed vaporizing liquid drops. Atom. Sprays 6, 353-376.

Joseph, D. D., Belanger, J. \& Beavers, G. S. 1999 Breakup of a liquid drop suddenly exposed to a high-speed airstream. Intl J. Multiphase Flow 25, 1263-1303.

Kelvin, LORD 1871 Hydrokinetic solutions and observations. Phil. Mag. 42, 362-377.

Lasheras, J. C. \& Hopfinger, E. J. 2000 Liquid jet instability and atomization in a coaxial gas stream. Annu. Rev. Fluid Mech. 32, 275-308.

Lasheras, J. C., Villermaux, E. \& Hopfinger, E. J. 1998 Break-up and atomization of a round water jet by a high-speed annular air jet. J. Fluid Mech. 357, 351-379.

LefebVRe, A. H. 1989 Atomization and Sprays. Hemisphere.

LiN, S. P. 1995 Regimes of jet breakup and breakup mechanisms (mathematical aspects). In Recent Advances in Spray Combustion: Spray Atomization and Drop Burning Phenomena (ed. K. K. Kuo), vol. 1, pp. 137-160.

Lin, S. P. \& Lian, Z. W. 1989 Absolute instability of a liquid jet in a gas. Phys. Fluids A 1, 490-493.

Liv, Z. \& Reitz, R. D. 1997 An analysis of the distortion and breakup mechanisms of high speed liquid drops. Intl J. Multiphase Flow 23, 631-650.

Lorenzetto, G. E. \& Lefebvre, A. H. 1977 Measurements of drop size on a plain-jet airblast atomizer. AIAA J. 15, 1006-1010.

Mansour, A. \& Chigier, N. 1991 Dynamic behavior of liquid sheets. Phys. Fluids A 3, 2971-2980.

Marmottant, P. 2001 Atomisation d'un jet liquide par un courant gazeux. Thèse de Doctorat, Institut National Polytechnique, Grenoble, France.

Nukiyama, G. E. \& Tanasawa, Y. 1939 Experiments on the atomization of liquids by means of air streams, parts III and IV. Trans. Soc. Mech. Eng. Japan 5(18), 63-75.

Pilch, M. \& ERdman, C. A. 1987 Use of break-up time data and velocity history data to predict the maximum size of stable fragments for acceleration-induced break-up of a liquid drop. Intl J. Multiphase Flow. 13, 741-757.

RAYNAL, L. 1997 Instabilité et entrainment à l'interface d'une couche de mélange liquid-gaz. Thèse de Doctorat, Université Joseph Fourier, Grenoble, France.

Reitz, R. D. \& Bracco, F. V. 1982 Mechanism of atomization of a liquid jet. Phys. Fluids. 25, $1730-1742$.

Rizkalla, A. A. \& Lefebvre, A. H. 1975 The influence of air and liquid properties on air blast atomization. Trans. ASME: J. Fluids Engng 97, 316-320.

TAYLOR, G. I. 1950 The instability of liquid surfaces when accelerated in a direction perpendicular to their planes, Part I. Proc. Roy. Soc. A 201, 192-196.

TAYLOR, G. I. 1963 Generation of ripples by wind blowing over a viscous liquid. In The Scientific Papers of G.I. Taylor (ed. G. K. Batchelor), vol. 3, pp. 244-254. Cambridge University Press.

Villermaux, E. 1998 Mixing and spray formation in coaxial jets. J. Prop. Power 14, 807-817. 